\title{
Near-Field Energy Transfer Using Nanoemitters For Optoelectronics
}

\author{
Burak Guzelturk and Hilmi Volkan Demir*
}

Effective utilization of excitation energy in nanoemitters requires control of exciton flow at the nanoscale. This can be readily achieved by exploiting nearfield nonradiative energy transfer mechanisms such as dipole-dipole coupling (i.e., Förster resonance energy transfer) and simultaneous two-way electron transfer via exchange interaction (i.e., Dexter energy transfer). In this feature article, we review nonradiative energy transfer processes between emerging nanoemitters and exciton scavengers. To this end, we highlight the potential of colloidal semiconductor nanocrystals, organic semiconductors, and twodimensional materials as efficient exciton scavengers for light harvesting and generation in optoelectronic applications. We present and discuss unprecedented exciton transfer in nanoemitter-nanostructured semiconductor composites enabled by strong light-matter interactions. We elucidate remarkably strong nonradiative energy transfer in self-assembling atomically flat colloidal nanoplatelets. In addition, we underscore the promise of organic semiconductor-nanocrystal hybrids for spin-triplet exciton harvesting via Dexter energy transfer. These efficient exciton transferring hybrids will empower desired optoelectronic properties such as long-range exciton diffusion, ultrafast multiexciton harvesting, and efficient photon upconversion, leading to the development of excitonic optoelectronic devices such as exciton-driven light-emitting diodes, lasers, and photodetectors.

\section{Introduction}

Optoelectronics is a key technology that has revolutionized numerous fields ranging from energy generation to

Dr. B. Guzelturk, ${ }^{[+]}$Prof. H. V. Demir

Department of Electrical and Electronics Engineering Department of Physics

UNAM - Institute of Materials Science

and Nanotechnology

Bilkent University

Ankara 06800, Turkey

E-mail: volkan@bilkent.edu.tr, hvdemir@ntu.edu.sg

Prof. H. V. Demir

Luminous! Center of Excellence for Semiconductor

Lighting and Displays

School of Electrical and Electronic Engineering

School of Physical and Mathematical Sciences

School of Materials Science and Engineering

Nanyang Technological University

Nanyang Avenue, Singapore 639798, Singapore

${ }^{[+]}$Present address: Stanford Institute for Materials and Energy Sciences, SLAC National Accelerator Laboratory, Menlo Park, California, 94025, USA

DOI: 10.1002/adfm.201603311 telecommunications. Conventional optoelectronics relies on high-temperature, gas-phase, epitaxy-grown semiconductors such as III-V compounds, ${ }^{[1,2]}$ which make a mature materials technology. However, high-thermal budget, high-cost, and a limited set of substrates, which are CMOS-incompatible, hamper their use in versatile platforms, including flexible surfaces and large-area applications. Besides, the last decade has witnessed the rise of alternative low-dimensional semiconductor materials such as colloidal semiconductor nanocrystals, ${ }^{[3]}$ organic semiconductors $^{[4,5]}$ and more recently, two-dimensional (2D) semiconductors. ${ }^{[6]}$ These materials offer advantages over conventional semiconductors thanks to their low cost and low thermal budget growth, solution processability, and roll-to-roll fabrication on arbitrary substrates on a large scale. These materials are expected to impact a broad range of applications in optoelectronics and electronics.

In bulk and weakly confined semiconductors, the excited electronic state is typically in the form of free electron-hole pairs due to weak Coulomb interaction energy $(\sim 10 \mathrm{meV}) \cdot{ }^{[7]}$ On the other hand, in low-dimensional nanoemitters, the excited state is essentially in the form of strongly bound electron-hole pairs (i.e., excitons) with large Coulomb interaction energy $(10 \mathrm{meV})$ thanks to strong quantum confinement ${ }^{[8]}$ and large dielectric screening, ${ }^{[9,10]}$ allowing for strong light-matter interactions. Thus, in these nanoemitters, it becomes central to control excitonic interactions including exciton transfer, diffusion, trapping, dissociation, annihilation, and radiative recombination to accomplish the desired photonic properties and maximize optoelectronic performance.

As it naturally happens in photosynthetic light-harvesting complexes, efficient and directed exciton flow is highly desired in semiconductor nanostructures. To this end, mastering exciton flow at the nanoscale through near-field nonradiative energy transfer has proven vital to accomplish efficient light generation and light utilization using nanoemitters and their hybrid nanostructures. ${ }^{[7,11-15]}$ In this feature article, we highlight recent developments in the field of energy transfer materials for optoelectronics. We review new insights on the near-field nonradiative transfer in excitonic nanoemitter systems. Our main focus is on hybrid systems comprising colloidal nanocrystals, and 2D and organic semiconductors. Also, 
we explore the potential optoelectronic applications of the energy transferring hybrids together with their prospects in sensing and bio-imaging.

\section{Nonradiative Energy Transfer Mechanisms: FRET vs Dexter}

Excitons can be nonradiatively transferred from one molecule to another through near-field interactions such as dipoledipole coupling and simultaneous two-way electron transfer via exchange interaction, which are known as Förster resonance energy transfer $\left(\right.$ FRET) ${ }^{[16]}$ and Dexter energy transfer, ${ }^{[17]}$ respectively. Figure 1 schematically illustrates these processes between an exemplary donor (e.g., colloidal quantum dot)-acceptor (e.g., pentacene molecule) pair. In these energy transfer processes, the excited donor $\left(D^{*}\right)$ relaxes into its ground-state (D) while the excited state energy has been transferred to the acceptor $\left(A^{*}\right)$, which was in its ground-state (A) before the transfer occurred. The overall energy transfer process can be represented by $D^{*}+A \stackrel{k_{E T}}{\rightarrow} D+A^{*}$. These processes are called nonradiative energy transfers because they do not involve the emission or absorption of a photon.

FRET is a near-field dipole-dipole coupling process where reactive electromagnetic near-field of an oscillating transition dipole in the donor induces a transition dipole in the acceptor (see Figure 1a). Under a weak interaction limit, the FRET rate is directly related to the square of the Coulombic dipole-dipole interaction energy $\left(E_{\text {exc }}\right)^{[18]}$ :

$k_{E T}(F R E T) \approx\left(E_{e x c}\right)^{2}=\left(\frac{\vec{p}_{D^{*} \rightarrow D} \cdot \vec{p}_{A \rightarrow A^{*}}}{\mathrm{~d}^{3}}\right)^{2}$

where $k_{E T}$ (FRET) is the FRET rate; $\vec{p}_{D^{*} \rightarrow D}$ and $\vec{p}_{A \rightarrow A^{*}}$ are the transition dipole moments of the $D^{*} \rightarrow D$ and $A \rightarrow A^{*}$ transitions, respectively; and $\mathrm{d}$ is the center-to-center separation distance between the donor and the acceptor. In a more conventional form, the FRET rate can be expressed as follows:

$k_{E T}(F R E T) \approx \frac{\mathrm{f}_{D^{*} \rightarrow D} \times \kappa^{2} \times J_{D A}\left(\mathrm{f}_{A \rightarrow A^{*}}\right)}{\mathrm{d}^{6}}$

Here, $f_{D^{*} \rightarrow D}$ and $f_{A \rightarrow A^{*}}$ are the oscillator strengths of the transitions denoted in the subscript, $\kappa^{2}$ is the dipole orientation factor, $J_{D A}$ is the spectral overlap integral between the donor emission and the acceptor absorbance, which implies the existence of energetically resonant states in the acceptor molecule that match the energy of the excited state in the donor (if $D^{*} \rightarrow D+h v$, then $A+h v \rightarrow A^{*}$ should be possible). Also, $J_{D A}$ is a function of $\mathrm{f}_{A} \rightarrow A_{A^{*}}$ because it is related to the extinction coefficient of the acceptor. Thus, the FRET rate necessarily depends on the oscillator strength of both the donor and the acceptor. Because of this, only singlet states, which have nonzero oscillator strength, can be transferred via FRET. One important distinction is that although the FRET rate is directly related to the donor's decay rate, FRET efficiency is not. FRET efficiency essentially depends on the distance between the donor-acceptor pair and the acceptor's absorption oscillator

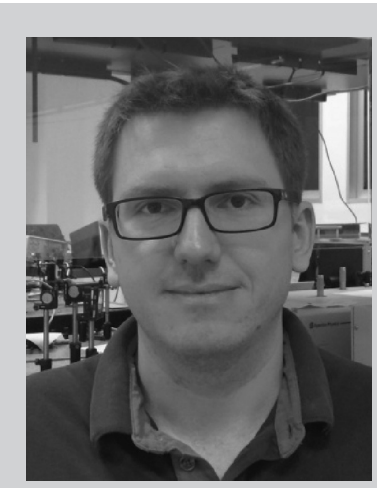

Burak Guzelturk received his B.S. (2009), M.S. (2011), and Ph.D. (2016) degrees in the Department of Electrical and Electronics Engineering at Bilkent University. $\mathrm{He}$ pursued his Ph.D. under the guidance of Prof. Hilmi Volkan Demir. Currently, he is a postdoctoral research fellow at Stanford University and the SLAC National Accelerator

Laboratory in the United States. To date, he has been awarded by the IEEE Photonics Society, the European Technology Platform Photonics21, the German Federal Ministry of Education and Research, and the SPIE for his $\mathrm{PhD}$ study. His research interests include understanding excitonic properties in nanoemitters via ultrafast spectroscopy, and tailoring these nanomaterials towards efficient optoelectronics.

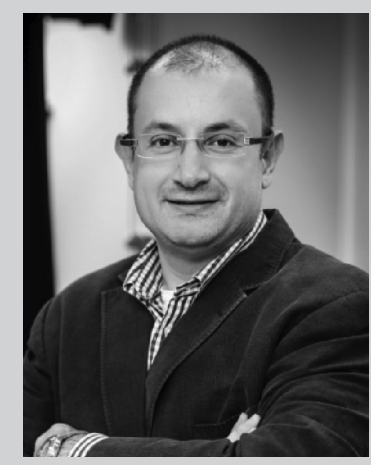

Hilmi Volkan Demir received his M.S. and Ph.D. degrees in electrical engineering from Stanford University in 2000 and 2004, respectively. In 2004, he joined Bilkent University as Professor at the Department of ElectricalElectronics Engineering and the Department of Physics. $\mathrm{He}$ is the Director of the National Nanotechnology Research Center (UNAM) and the Institute of Materials Science and Nanotechnology at Bilkent University. He is concurrently Nanyang Professor at the School of Electrical Electronic Engineering and Physical and Mathematical Sciences, NTU, and the Director of the LUMINOUS! Center of Excellence for Semiconductor Lighting and Displays. His current research interests include energysaving LEDs for quality lighting, the science of excitonics for high-efficiency light harvesting, and nanocrystal optoelectronics.

strength. Furthermore, respective orientation of the donor and the acceptor dipoles is important. For randomly oriented dipoles, the dipole orientation factor $\left(\kappa^{2}\right)$ is $2 / 3$, and it reaches its maximum value of 4 when the transition dipoles are collinear. For the donors with a photoluminescence quantum yield (QY) less than unity, the FRET rate can be related as follows:

$$
\begin{aligned}
& k_{\text {decay }}=k_{\text {rad }}+k_{\text {nonrad }}=\frac{k_{\text {rad }}}{Q Y} \\
& k_{E T}(F R E T) \approx \frac{k_{\text {decay }} \times Q Y \times \kappa^{2} \times J_{D A}}{\mathrm{~d}^{6}}
\end{aligned}
$$


FRET

\section{(a)}

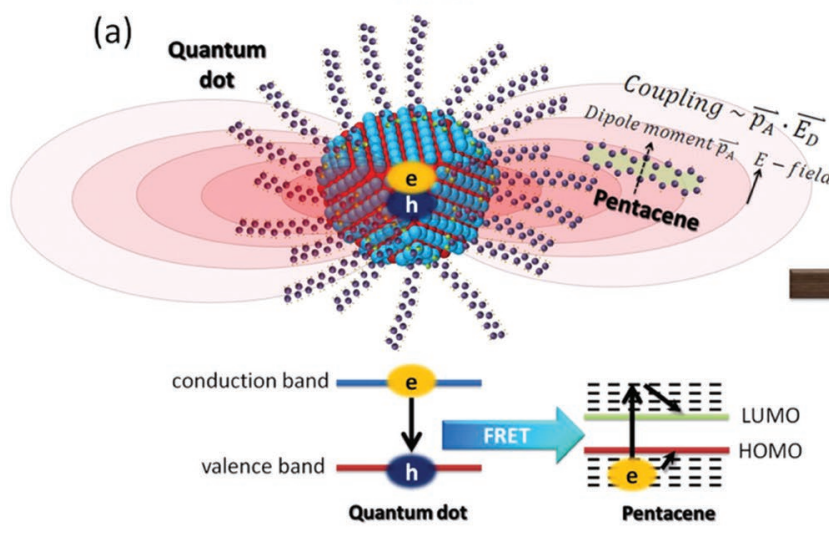

Dexter Energy Transfer

(b)

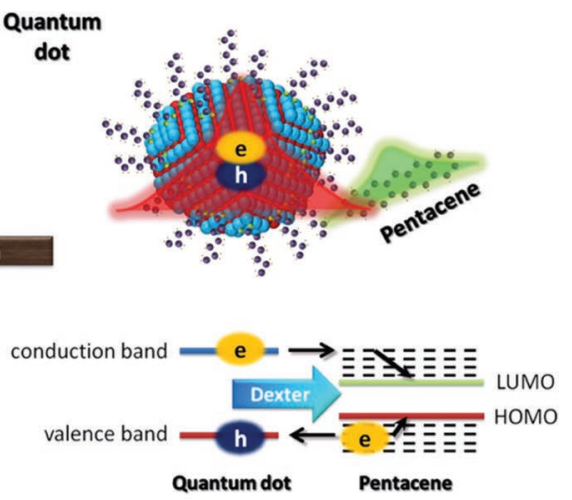

Figure 1. Schematic illustration of (a) Förster resonance energy transfer (FRET) and (b) Dexter energy transfer. (a) The reactive near-field electromagnetic field of an oscillating transition dipole in a donor (e.g., CdSe quantum dot) induces a transition dipole in an acceptor (e.g., pentacene). The energy band diagram (below) depicts the resonant transfer of the excitons. (b) A simultaneous electron exchange process (Dexter energy transfer) leads to the transfer of excitons from a donor into an acceptor thanks to the overlapping charge wavefunctions. The energy band diagram highlights Dexter energy transfer that occurs through simultaneous electron exchange interaction.

FRET has a distance scaling that intrinsically depends on the geometry of the acceptor. For point-like dipole pairs, the "classic" $d^{-6}$ scaling factor exists. For one-dimensional (e.g., nanowire), two-dimensional (e.g., quantum well) and threedimensional (e.g., bulk semiconductor) acceptors, the distance scaling term ranges from $d^{-5}, d^{-4}$, and $d^{-3}$, respectively. ${ }^{[19,20]}$ Moreover, a critical distance between donor-acceptor pair is defined, which is commonly known as the Förster radius $\left(R_{0}\right)$. FRET efficiency becomes $50 \%$ when the donor-acceptor separation distance is equal to $R_{0}$, which is expressed as ${ }^{[21]}$

$$
R_{0}=\left(\frac{9000 \ln 10 \kappa^{2} Q Y J_{D A}}{N_{A} 128 \pi^{5} n^{4}}\right)^{\frac{1}{6}}
$$

where $N_{A}$ is Avagadro's number and $n$ is the refractive index. The Förster radius for typical donor-acceptor pairs is ca. 3-7 nm. ${ }^{[18,21]}$ Recently, longer Förster radii $(>10 \mathrm{~nm})$ could be realized by using acceptor materials that have strong light absorption, which is discussed in this feature article. Table 1 highlights effective Förster radii for the recently explored hybrid

Table 1. Förster radii for different donor-acceptor pairs commonly consisting of colloidal quantum dots, nanoplatelets, 2D materials, and organic semiconductors.

\begin{tabular}{|c|c|c|c|c|}
\hline Donor & Donor emission peak & Donor PL QY & Acceptor & Förster radius $\left(R_{0}\right)$ \\
\hline $\mathrm{CdSe} / \mathrm{CdS}$ core/shell quantum dot & $580 \mathrm{~nm}$ & $\mathrm{~N} / \mathrm{A}$ & Single layer graphene & $11.5 \mathrm{~nm}^{[22]}$ \\
\hline Rhodamine dye & $650 \mathrm{~nm}$ & $\mathrm{~N} / \mathrm{A}$ & Single layer graphene & $15 \mathrm{~nm}^{[23]}$ \\
\hline InGaN quantum well capped with $\mathrm{GaN}$ & $440 \mathrm{~nm}$ & $\mathrm{~N} / \mathrm{A}$ & Graphene & $16 \mathrm{~nm}^{[24]}$ \\
\hline InGaN quantum well capped with $\mathrm{GaN}$ & $405 \mathrm{~nm}$ & $\mathrm{~N} / \mathrm{A}$ & Graphene oxide & $6.4 \mathrm{~nm}^{[25]}$ \\
\hline $\mathrm{CdSe} / \mathrm{CdS}$ core/shell quantum dot & $580 \mathrm{~nm}$ & $70 \%$ & Few layer graphene oxide & $7.5 \mathrm{~nm}^{[26]}$ \\
\hline $\mathrm{CdSe} / \mathrm{CdS}$ core/shell quantum dot & $620 \mathrm{~nm}$ & $\mathrm{~N} / \mathrm{A}$ & Single layer $\mathrm{MoSe}_{2}$ & $15.4 \mathrm{~nm}^{[27]}$ \\
\hline $\mathrm{CdSe} / \mathrm{ZnS}$ core/shell quantum dot & $525 \mathrm{~nm}$ & $\mathrm{~N} / \mathrm{A}$ & Single layer $\mathrm{SnS}_{2}$ & $4-5 \mathrm{~nm}^{[28]}$ \\
\hline $\mathrm{CdSe} / \mathrm{CdSeS} / \mathrm{CdS}$ core/shell quantum dot & $605 \mathrm{~nm}$ & $50 \%$ & Singe or few layer $\mathrm{MoS}_{2}$ and $\mathrm{WS}_{2}$ & $5 \mathrm{~nm}^{[29]}$ \\
\hline \multirow[t]{2}{*}{$\mathrm{CdSe} / \mathrm{CdZnS}$ core/shell quantum dot } & $635 \mathrm{~nm}$ & $80 \%$ & Single layer $\mathrm{MoS}_{2}$ & $19 \mathrm{~nm}$ \\
\hline & & & Bulk $\mathrm{MoS}_{2}$ & $11 \mathrm{~nm}^{[30]}$ \\
\hline 4 monolayer $(\mathrm{ML}) \mathrm{CdSe}$ nanoplatelet & $513 \mathrm{~nm}$ & $30 \%$ & $4 \mathrm{ML}$ CdSe nanoplatelet & $13.5 \mathrm{~nm}^{[31]}$ \\
\hline CdZnS/ZnS core/shell quantum dot & $450 \mathrm{~nm}$ & $38 \%$ & $4 \mathrm{ML}$ CdSe nanoplatelet & $9.5 \mathrm{~nm}^{[32]}$ \\
\hline $4 \mathrm{ML}$ CdSe nanoplatelet & $513 \mathrm{~nm}$ & $40 \%$ & $5 \mathrm{ML}$ CdSe nanoplatelet & $10.8 \mathrm{~nm}^{[33]}$ \\
\hline $\mathrm{CdSe} / \mathrm{ZnS}$ core/shell quantum dot & $570 \mathrm{~nm}$ & $90 \%$ & Bulk silicon & $5.9 \mathrm{~nm}^{[34]}$ \\
\hline Si nanocrystals & $627 \mathrm{~nm}$ & $30 \%$ & Silicon nanowire & $6.2 \mathrm{~nm}^{[35]}$ \\
\hline Polyfluorene & $425 \mathrm{~nm}$ & $50 \%$ & $\mathrm{CdSe} / \mathrm{CdZnS} / \mathrm{ZnS}$ core/shell quantum dot & $5 \mathrm{~nm}^{[36]}$ \\
\hline $\mathrm{Alq}_{3}$ & $530 \mathrm{~nm}$ & NA & Common organic semiconductors & $2-4 \mathrm{~nm}^{[37]}$ \\
\hline Flrpic & $480 \mathrm{~nm}$ & $>90 \%$ & $\mathrm{CdSe} / \mathrm{CdZnS} / \mathrm{ZnS}$ core/shell quantum dot & $6 \mathrm{~nm}^{[38]}$ \\
\hline TCTA & $390 \mathrm{~nm}$ & $\mathrm{~N} / \mathrm{A}$ & $\mathrm{CdSe} / \mathrm{CdS} / \mathrm{ZnS}$ core/shell quantum dot & $5 \mathrm{~nm}^{[38]}$ \\
\hline
\end{tabular}


systems such as quantum dot-2D material, quantum dot-nanoplatelet, and organic semiconductor-quantum dot.

Dexter energy transfer is the other common near-field nonradiative energy transfer process. Dexter energy transfer relies on the wavefunction (charge orbital) overlap between the donor and the acceptor species. It can be considered as simultaneous tunneling of electron and hole from the donor into the acceptor. Therefore, for Dexter energy transfer to take place, donor and acceptor molecules should be very close to each other (surface-to-surface separation distance generally $\leq 1 \mathrm{~nm}$ ). Also, wavefunction overlap should exist for the orbitals of interacting initial and final states of the electron and hole, respectively.

In Dexter energy transfer, which is a short-range energy transfer process, the transfer rate can be expressed in the form of an exponential decay:

$k_{E T}($ Dexter $)=\mathrm{K} \times J_{D A}^{\text {norm }} D A \times e^{\left(\frac{-2 d}{L}\right)}$

Here $k_{E T}$ (Dexter) is the Dexter transfer rate, $\mathrm{K}$ is a factor related to the specific orbital interactions, $J_{D A}^{\text {norm }}$ is the normalized spectral overlap integral term, $d$ is the center-to-center distance between the donor and the acceptor, and $L$ is the characteristic wave function decay length (e.g., van der Waals radius). $J_{D A}^{\text {norm }}$ is different from the $J_{D A}$ that is used in the FRET calculation because the acceptor's oscillator strength is not important for Dexter energy transfer. Only the normalized absorbance of the acceptor and the normalized emission of the donor are considered to calculate the normalized spectral overlap $\left(J_{D A}^{\text {norm }}\right)$, which should satisfy the resonance condition.

Figure $1 \mathrm{~b}$ schematically illustrates Dexter energy transfer from an exemplary donor CdSe quantum dot into an exemplary acceptor pentacene molecule. Organic ligands of the quantum dot are intentionally chosen to be shorter (Figure 1b) to highlight the need for intimate coupling and the wavefunction overlap between the species. Different from FRET, oscillator strength of the donor does not play a role in Dexter energy transfer. Therefore, energy transfer from non-emissive triplet states become feasible via Dexter energy transfer. Table 2 summarizes the similarities and differences between FRET and Dexter energy transfer processes.

\section{Exciton Funneling for Light Harvesting}

Funneling excitons from a strongly absorbing material into a medium with large electrical conductivity has been established as a promising strategy to efficiently convert light into electricity. ${ }^{[17,39]}$ Previously, FRET-based exciton funneling schemes ${ }^{[40]}$ were suggested for photosensing and photovoltaics as promising alternatives to photoinduced charge injectionbased photosensitization. ${ }^{[41]}$ Charge injection/transfer processes could only happen for very short separation distances $(\leq 1 \mathrm{~nm})$, thus, FRET-based exciton harvesting, which enable energy transfer at longer distances $(\geq 5 \mathrm{~nm})$, can potentially be more efficient.

In Figure 2a, we illustrate a model light-harvesting nanostructure consisting of a strongly light-absorbing sensitizer (e.g., colloidal quantum dots) that is interfaced with an exciton sink layer (e.g., bulk silicon), which exhibits high electrical conductivity. Photogenerated excitons in the sensitizer layer can be transferred to the sink via near-field nonradiative energy transfer. The resonantly transferred excitons contribute to the generation of photocurrent in the sink layer. For this, electron-hole pairs in the sink must be dissociated into free carriers either by applying an external bias (see Figure $2 b$ ) or by creating hetero- or homo-interfaces with staggered band alignment such as p-n junction (see Figure 2c).

Ideally, sensitizer materials should combine large oscillator strength and high photoluminescence quantum yield together. Commonly, colloidal quantum dots and organic semiconductors have been considered as the sensitizer materials to harvest the photogenerated excitons. As exciton sink layers, materials with high electrical mobility are desired, such as bulk or nanostructured (i.e., quantum wires and wells) semiconductors. Also, 2D semiconductors and colloidal nanoplatelets have recently emerged as promising exciton sinks. Previously, exciton funneling nanostructures have proven to be exciting for photodetectors and photovoltaics. ${ }^{[42-44]}$

Table 2. Similarities and differences between FRET and Dexter energy transfer processes.

\begin{tabular}{|c|c|c|}
\hline & FRET & Dexter energy transfer \\
\hline Transfer mechanism & Dipole-dipole coupling & Wavefunction (electron orbital) overlap \\
\hline \multirow[t]{4}{*}{ Distance scaling } & $d^{-6}$ (for point-like dipoles) & \\
\hline & $d^{-5}$ (for 1D acceptor, e.g., nanowire) & $e^{\left(\frac{-2 d}{L}\right)}$ \\
\hline & $d^{-4}$ (for 2D acceptor, e.g., quantum well) & \\
\hline & $d^{-3}$ (for 3D acceptor, e.g., bulk) & \\
\hline Resonance condition & Yes, requires non-zero spectral overlap term $\left.U_{D A}\right)$ & Yes, requires non-zero normalized spectral overlap $\left(J_{D A}^{\text {norm }}\right)$ \\
\hline Effective distance & 2-10 nm (long-range) & $\leq 1 \mathrm{~nm}$ (short-range) \\
\hline Type of the excitons & Singlet-singlet & Singlet-singlet, triplet-triplet \\
\hline $\begin{array}{l}\text { Dependence of the energy transfer rate on the } \\
\text { quantum yield of the donor }\end{array}$ & Yes, indirectly through total decay rate of the donor & Yes, indirectly through total decay rate of the donor \\
\hline Dependence on transition dipole strength & Yes & No \\
\hline
\end{tabular}




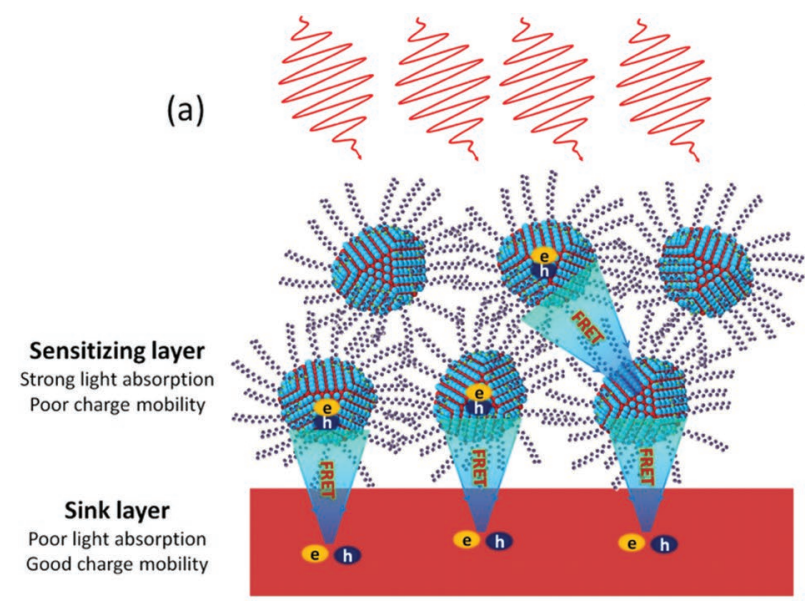

Photocurrent generation from exciton sink layers

(b)

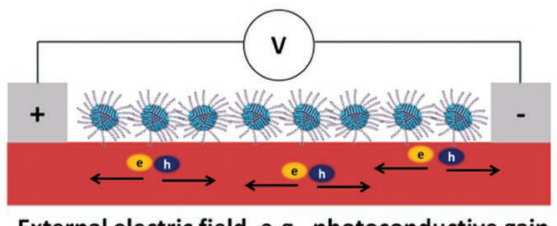

External electric field, e.g., photoconductive gain

(c)

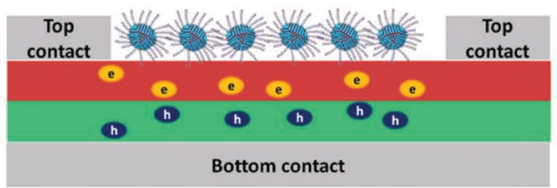

Interface with staggered band alignment, e.g., p-n junction

Figure 2. (a) A hybrid platform for exciton funneling from a sensitizer layer (e.g., colloidal quantum dots) into an exciton sink layer (e.g., silicon). The sensitizer has strong and broadband light absorption and the sink layer possesses good electrical conductivity. Strategies for photocurrent generation in exciton-transferring sensitizer-sink structures: (b) applying external electric field and (c) creating interfaces with staggered band alignment, i.e., p-n junction.

\section{Sensitization of Bulk and Nanostructured Semiconductors}

Bulk semiconductors such as silicon and gallium arsenide are typically used for photodetectors and photovoltaics; especially silicon dominates the photovoltaics industry. However, photovoltaic devices, e.g., silicon solar cells, generally suffer from poor spectral utilization of sunlight. For example, UVblue ${ }^{[45]}$ and NIR-IR ${ }^{[46]}$ portions of the solar spectrum cannot be efficiently converted into electricity by silicon solar cells. To overcome the poor spectral light utilization problem, external sensitization of silicon has been explored via photon downconversion ${ }^{[46]}$ that can also harness multiple-exciton generation in the nanoemitters. ${ }^{[4]}$ To date, among different sensitization schemes, photon down conversion through radiative energy transfer using light-emitting phosphors has been intensively investigated. ${ }^{[48-50]}$ Notably, nanostructured solar cell architectures showed enhanced sensitization thanks to the increased light trapping. ${ }^{[45,51]}$ However, sensitizing silicon through radiative energy transfer is inherently restrained due to the limited coupling of the emitted photons to the active medium of the solar cell. Also, multiple radiative recombination and absorption processes lead to significant losses due to intrinsic nonradiative decay channels in the sensitizer materials.

Alternatively, nonradiative energy transfer could be employed to directly transfer photogenerated electron-hole pairs into bulk and nanostructured semiconductors without emission of a photon by the sensitizer. In the 1970s, Chance, Prock, and Silbey theoretically predicted nonradiative energy transfer into various metals and semiconductors, including silicon and gallium arsenide. ${ }^{[52]}$ Alivisatos et al. experimentally demonstrated the spectroscopic evidence of nonradiative energy transfer from an organic molecule pyrene into bulk silicon. ${ }^{\text {[53] }}$ Ref. [54] also uncovered nonradiative energy transfer from the organic molecule pyrazine into direct bandgap GaAs. After these early studies, more recently, Madhukar et al. proposed that colloidal quantum dots would be promising sensitizers for silicon through exciton funneling via nonradiative energy transfer thanks to their tunable and bright emission, spectrally broad absorption features, and increased environmental stability. ${ }^{[5]}$ In Ref. [55], FRET from near-IR-emitting PbS quantum dots into adjacent InGaAs quantum wells was demonstrated, where the latter acted as an efficient exciton sink and charge transport channel. After this work, Ref. [56] observed FRET from visibleemitting $\mathrm{CdSe} / \mathrm{CdS}$ quantum dots into GaAs quantum wells, where near-field coupling strength was considerably enhanced by realizing nanohole arrays on the sink material surface. This scheme resulted in 6-fold enhancement of the photocurrent thanks to the intimate integration and the resulting enhanced FRET. Inspired by these works, different quantum dot sensitizers and nanostructured semiconductor sinks were also tested. For example, water soluble CdTe quantum dots were integrated with a GaAs quantum well nanohole array, which led to 3-fold photocurrent enhancement. ${ }^{[57]}$ InAs quantum dots were used to enhance photo-transistors of GaAs quantum wells. ${ }^{[58]}$ InGaN quantum wells and CdSe nanowires were also investigated as exciton sinks and charge transport channels when combined with $\mathrm{CdSe} / \mathrm{ZnS}^{[59]}$ and $\mathrm{CdSe} / \mathrm{CdS}^{[00]}$ core/shell quantum dots.

To understand the physical origin of the enhanced photocurrent in these hybrid systems, Ref. [43] employed timeresolved photocurrent measurements using a hybrid structure consisting of $\mathrm{PbS}$ quantum dots decorated on a laterallypatterned silicon substrate. The time-resolved photocurrent measurements strongly suggested the contribution of resonantly transferred excitons to the total photocurrent. Furthermore, fluorescence spectroscopy measurements on a monolayer CdSe/ZnS quantum dot film placed on bulk silicon substrate revealed the efficiency of nonradiative energy transfer into silicon. ${ }^{[34]}$ Considering Förster-like dipole-dipole coupling, near-field energy transfer efficiency was found to be $65 \%$ at an effective donor-acceptor distance of $4.4 \mathrm{~nm}$. The resulting effective Förster radius was estimated to be $5.9 \mathrm{~nm}$ (see Table 1).

Recently, a temperature-dependent investigation of FRET from a CdSe/ZnS quantum dot film into bulk silicon revealed a strong dependence on the temperature. ${ }^{[61]}$ In this quantum 
dot-silicon hybrid, FRET efficiencies and rates were observed to be considerably decreased at cryogenic temperatures compared to those at room temperature. Previously, in the case of quantum dot-based donors, FRET efficiency has been found to be typically constant as a function of temperature. ${ }^{[62,63]}$ Different from previously studied quantum dot donor-direct bandgap acceptor systems, when silicon was used as an acceptor material, the observed strong temperature dependency of the FRET efficiency originated mainly from a highly temperature-dependent absorption cross-section of silicon. Since light absorption is a phonon-assisted process (see Figure 3a), the absorption cross-section of silicon is considerably reduced as the temperature is decreased. Furthermore, the temperaturedependent photoluminescence quantum yield of the quantum dots also played a role in the observed changes in the kinetics.

Previously, Dexter et al. predicted that FRET into an indirect bandgap semiconductor could be made more efficient, if the dipole emitter would be placed very close to the surface of the acceptor. This would arise since the wave vector of the dipole near-field $\left(k_{\text {nearfeld }}=h / d\right)$ would be much larger than the wave vector of the emitted photon in the far field $\left(k_{\text {photon }}=h / \lambda\right)$ since $d \ll \lambda$, where $h$ is Planck's constant, $d$ is the separation distance, and $\lambda$ is the wavelength of light. ${ }^{[64]}$ Therefore, as shown in Figure 3a, nonvertical excitation via FRET into an indirect bandgap could be possible thanks to the large wave vector of the dipole near-field ( $\left.k_{\text {nearfield}}\right)$. However, the separation distance should be $\leq 2 \mathrm{~nm}$ to realize a near-field wave vector that can cover a significant portion of the Brillouin zone of the indirect bandgap semiconductor (e.g., silicon). ${ }^{[53]}$ In the case of CdSe/ $\mathrm{ZnS}$ quantum dots, the separation distance was larger than $4 \mathrm{~nm}$ considering the radius of the quantum dots, organic capping ligands, and the native oxide layer. Because of this, in the quantum dot-silicon hybrid, FRET essentially required the assistance of phonons. ${ }^{[61]}$ More importantly, we realized that when the temperature was increased to above room temperature $(290$ to $370 \mathrm{~K})$, FRET efficiency increased by more than 2-fold (see Figure 3b). This has been attributed to increased absorption in silicon at elevated temperatures due to stronger assistance of phonons and elongated exciton diffusion length in the quantum dot film, leading to a stronger FRET overall.

In addition to FRET, directed radiative energy transfer (RET) has been identified as an efficient way to boost light-harvesting in quantum dot-silicon hybrids. Since FRET is a near-field energy transfer process, its interaction range is quite limited. Therefore, using FRET, one could only efficiently transfer excitons that are formed in the closest quantum dot monolayer on top of silicon. To this end, Ref. [65] proposed to use ultrathin silicon nanomembranes (thickness $\approx 100-200 \mathrm{~nm}$ ) that exhibit photonic waveguiding modes, in which radiative emission of the quantum dots could couple effectively. Following this work, Ref. [66] showed that efficiencies of FRET and RET are highly sensitive to the emission wavelength of the sensitizing quantum dot due to the dispersion of the complex dielectric function of silicon. For the quantum dots emitting in the blue-yellow spectral range $\left(\lambda_{e m}<600 \mathrm{~nm}\right)$, FRET is stronger than RET. On the other hand, RET becomes stronger for the quantum dots emitting in the near-IR spectral range due to the small absorption cross-section of silicon that limits the dipole-dipole coupling strength. Figure $3 \mathrm{c}$ compares the calculated efficiencies of FRET and RET as a function of the peak emission wavelength of the quantum dots. Total energy transfer efficiency (FRET+RET) is also plotted on the same graph. Although FRET and RET have different efficiencies as a function of the nanoemitter's emission wavelength, the total energy transfer efficiency is almost constant $(-90 \%)$ for a monolayer of quantum dots on top of silicon. This suggests that although near-IR-emitting quantum dots could not be efficient FRET donors for silicon, RET could dominate the overall energy transfer. Therefore, RET could be highly advantageous for light-harvesting devices in addition to FRET.

Recently, several reports realized light sensors ${ }^{[67]}$ and photovoltaic devices ${ }^{[35,68]}$ using quantum dot-silicon hybrids that exhibited enhanced quantum efficiencies thanks to the efficient energy flow from the quantum dot sensitizers into silicon. Ref. [67] reported an almost two orders of magnitude enhancement of the photocurrent in a back-gated silicon membrane field-effect photo-transistor. FRET and RET were found to partially contribute to this large enhancement. Other factors such as change of the electrostatic potential of the channel surface and forward biasing of the metal-semiconductor junction due to exciton transfer were also found to play crucial roles.

Recently, Ref. [68] demonstrated ultrathin hybrid silicon solar cells (thickness $\approx 500 \mathrm{~nm}$ ) that exhibited power conversion efficiency of $1.60 \%$ when integrated with a $32 \mathrm{~nm}$-thick quantum dot sensitizer layer (corresponding to four quantum dot monolayers). The power conversion efficiency was $1.08 \%$ in the reference device that did not deploy a quantum dot sensitizer layer. Contribution of the energy transfer mechanisms (FRET and RET) to the enhanced photovoltaic performance was carefully assessed. Interestingly, the dominant contribution $(50 \%)$ was found to arise from the optical impedance matching, which arose due to the quantum dot layer acting as a graded refractive index layer between air and silicon. Contributions of FRET and RET to efficiency enhancement were found to be $13 \%$ and $37 \%$, respectively. ${ }^{[68]}$ Since the quantum dot layer thickness was relatively large, RET was more effective than FRET. Figure 3d depicts the calculated relative efficiencies of FRET and RET as a function of the separation distance between a monolayer quantum dot and silicon surface. Although FRET is dominant for short distances $(<10 \mathrm{~nm})$, RET is dominant larger separation distances.

Although photovoltaic performance could be enhanced by near-field energy transfer, one significant limitation to the device performance is the surface and bulk carrier recombination in poorly passivated silicon films. Since resonantly transferred electron-hole pairs in silicon are generated very close to the surface, these pairs could be easily lost due to effective nonradiative surface recombination processes. Thus, there is a strong need to employ well-passivated silicon surfaces to harvest the transferred electron-hole pairs more efficiently. Recently, Ref. [35] demonstrated a buried all-silicon solar cell architecture, which employed a low-temperature UV-ozone treated $\mathrm{n}$-type silicon nanowire array buried in a p-type silicon matrix. These solar cells exhibited considerable suppression of the surface and bulk recombination processes ${ }^{[69]}$ By decorating these silicon solar cells with fluorescent silicon nanocrystals, power conversion efficiencies as high as $12.9 \%$ were achieved with $20 \%$ enhancement as 
(a)

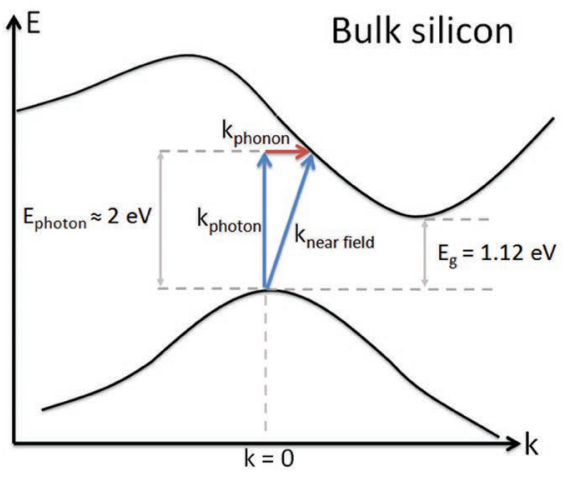

(c)

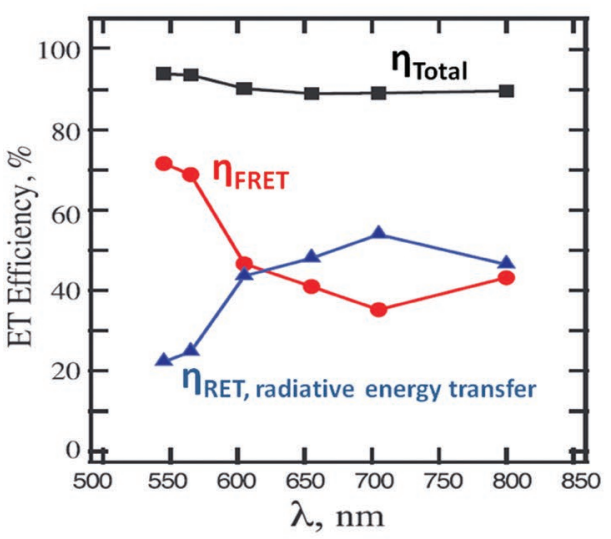

(b)
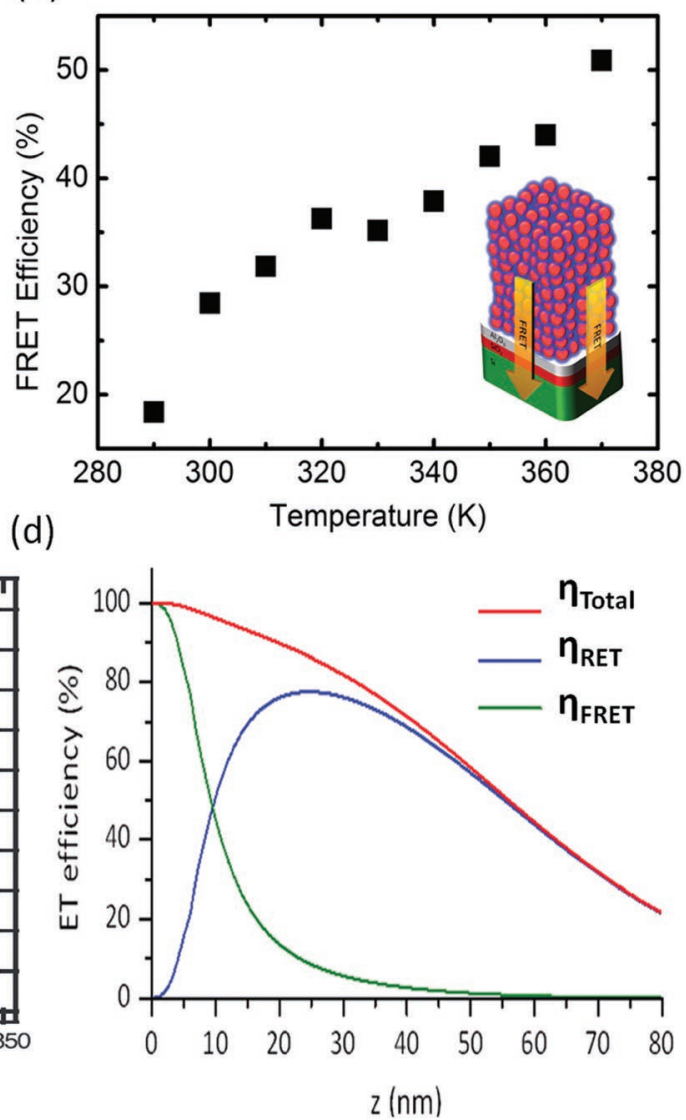

(e)
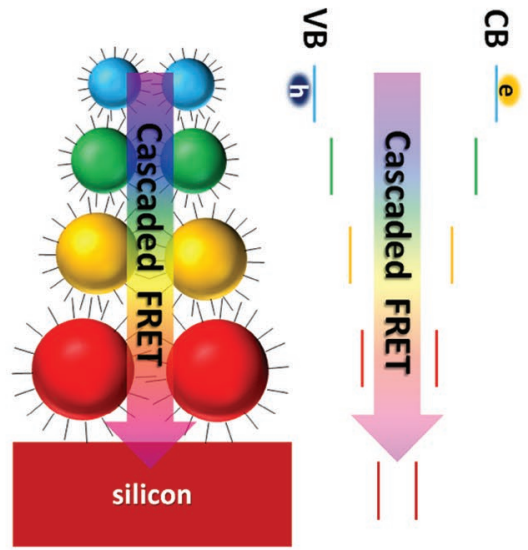

Figure 3. (a) Energy band diagram of bulk silicon. Optical absorption is phonon-assisted due to the indirect bandgap nature $\left(k_{\text {photon }}+k_{\text {phonon }}\right)$. Large wave vector of dipole near-field ( $k_{\text {nearfield }}$ ) of a nanoemitter on the surface of silicon could overcome the need for phonon assistance. (b) Temperaturedependent FRET efficiencies into silicon from few-layer-thick CdSe/ZnS quantum dots. Reproduced with permission. ${ }^{[61]}$ Copyright 2013 , American Chemical Society. (c) Efficiency of FRET, directed radiative energy transfer (RET) and total energy transfer (FRET+RET) for monolayer quantum dot films on silicon emitting at different spectral positions ranging from $550-800 \mathrm{~nm}$. Reproduced with permission. ${ }^{[66]}$ Copyright 2013, American Chemical Society. (d) FRET and RET efficiency for a quantum dot monolayer as a function of separation distance between the quantum dot layer and the silicon surface. Reproduced with permission. ${ }^{[68]}$ Copyright 2015, Royal Society of Chemistry. (e) Rainbow-like graded energy flow structure for multilayered quantum dot film for directed exciton funneling into silicon.

compared to the reference devices that did not incorporate any nanocrystal FRET-sensitizers. Suppression of the surface recombination in silicon allowed for the effective utilization of the transferred excitons. The enhancement was attributed to the near-field energy transfer from silicon nanocrystals into silicon nanowires. However, contributions of FRET and RET were not separately evaluated in these highly efficient photovoltaic devices. 
Another significant limitation of nonradiative energy transfer sensitization of silicon has been the utilization of single- or few-monolayer quantum dot layers. Considering the absorption cross-section of the conventional quantum dots $\left(10^{4}-10^{5} \mathrm{~cm}^{-1}\right),{ }^{[70,71]}$ thin quantum layers $(\approx 10 \mathrm{~nm})$ would lead to only $\approx 10 \%$ light absorption. Thus, thicker sensitizer layers $(\approx 100 \mathrm{~nm})$ are needed for efficient light-harvesting. However, excitons generated in the quantum dots that are away from the silicon surface cannot be effectively harvested using single bandgap quantum dots. This is because the excitons in the quantum dot film created further away from silicon cannot be easily transported towards silicon due to the limited exciton diffusion in quantum dot solids (typically 10-20 nm). ${ }^{[72,73]}$ To this end, use of graded energy gap quantum dot assemblies to funnel the excitons towards the silicon surfaces offers exciting possibilities. Figure 3e illustrates an exemplary graded gap quantum dot sensitizer layer that can achieve directed exciton flow towards the silicon surface. In our recent work, by employing a cascaded energy flow structure (green-emitting quantum dot/red-emitting quantum dot/silicon), we achieved more than 1.5-fold enhancement in the generation of electron-hole pairs in silicon as compared to bilayer control samples without energy gradient (red-emitting quantum dot/ red-emitting quantum dot/silicon and green-emitting quantum dot/green-emitting quantum dot/silicon). ${ }^{[74]}$ Later, Ref. [75] also investigated the potential of cascaded energy transfer using $\mathrm{CdSe} / \mathrm{ZnS}$ quantum dots into silicon, where they could achieve FRET efficiencies reaching 90\%. Previously, rainbowlike energy graded structure quantum dots were also employed for exciton-recycling[76] and light-harvesting in hybrid solar cells. ${ }^{[77-79]}$

\section{Two-Dimensional Materials as Efficient Exciton Sinks}

Two-dimensional materials, the so-called van der Waals materials, have recently emerged as highly promising for nanoelectronics, ${ }^{[6,80,81]}$ optoelectronics, ${ }^{\left[{ }^{[2-84]}\right.}$ and bio-sensing. ${ }^{[85,86]}$ Although 2D materials offer large electrical mobility and strong light-matter interactions, ${ }^{[80,82]}$ these materials are restricted by limited overall light absorption due to their ultrathin thicknesses (one to few monolayers). However, the absorption crosssection is very large in $2 \mathrm{D}$ materials. For example, a single layer graphene can absorb $2.3 \%$ of the light over a large spectrum (UV to IR), suggesting its huge absorption cross-section. To this end, sensitization of 2D materials by near-field energy transfer from adjacent nanoemitters has attracted increasing attention lately. ${ }^{[22,23,27,28,30,87-92]}$ Recent studies unveiled new physical insights on the near-field energy transfer and realized ultraefficient light-harvesting devices using 2D materials hybridized with nanoemitters.

Graphene was the first 2D material to be investigated as exciton sink from nearby nanoemitters via nonradiative energy transfer. ${ }^{[23,87,90,93]}$ Later, graphene derivatives such as graphene oxide ${ }^{[26,94,95]}$ and reduced graphene oxide, ${ }^{[26]}$ as well as amorphous carbon layers ${ }^{[96]}$ and carbon nanotubes, ${ }^{[97]}$ were considered for their capability as exciton accepting media for light-harvesting and bio-sensing applications. More recently, transition metal dichalcogenide (TMDC) monolayers were also investigated, such as $\mathrm{MoS}_{2},{ }^{[30,88,91]} \mathrm{MoSe}_{2},{ }^{[27]} \mathrm{WS}_{2},{ }^{[29]}$ and $\mathrm{SnS}_{2},{ }^{[28]}$ exhibiting strong exciton sinking capability comparable to that of graphene. As exciton-donating nanoemitters, colloidal semiconductor nanocrystals, ${ }^{[2,30,87]}$ nitrogen vacancy centers in diamond, ${ }^{[98]}$ and fluorescent dyes ${ }^{[99]}$ were typically employed. Here, we will focus more on the 2D material-nanocrystal hybrids and their prevailing photophysical properties, which make them exciting for numerous applications.

$\mathrm{CdSe} / \mathrm{ZnS}$ core/shell quantum dots that were deposited on top of a single-layer graphene exhibited strong photoluminescence quenching ( 70-fold). ${ }^{[87]}$ This quenching was well accounted for by strong near-field dipole coupling (i.e., FRET) into graphene. Also, the possibility of charge transfer leading to exciton dissociation at the graphene/quantum dot interface was ruled out due to the relatively thick shell of the quantum dots, which was shown to impede charge transfer. ${ }^{[100]}$ Using fluorescent dyes in a polymer matrix on top of a single-layer graphene, a more than 90 -fold increase in the fluorescence decay rate was reported, when the fluorescent dye is effectively $5 \mathrm{~nm}$ away from the graphene sheet. ${ }^{[23]}$ These early experimental reports suggested very strong exciton sink capability of graphene. Also, the effective Förster radius for graphene as an acceptor was estimated to be 11-16 $\mathrm{nm}^{[22-24]}$ (see Table 1). Thus, with exceptionally large Förster radius, graphene can empower long range $(>20 \mathrm{~nm})$ exciton transfer. This property of graphene has been attributed to its large absorption cross-section $\left(>10^{5} \mathrm{~cm}^{-1}\right)$. Although absolute absorption level of graphene is low $(2.3 \%$ over the visible and IR spectral region), its cross-section is large when considering graphene's only single-atom thickness. Furthermore, the gapless nature of graphene allows for frequency-independent efficient interband transitions, therefore making it an exciting candidate for an extremely broad range of nanoemitters.

TMDC monolayers were also investigated for their exciton sinking properties. For example, $\mathrm{MoS}_{2}$ led to strong modification of the fluorescence decay and large photoluminescence quenching of the nearby emitters, which were CdSe/CdZnS quantum dots. ${ }^{[30]}$ The dominant near-field interaction mechanism was found to be FRET, as understood by the sensitivity of the process to the spectral overlap between the emission of the quantum dots and the excitonic absorption of the $\mathrm{MoS}_{2}$ monolayer. ${ }^{[30]}$ An effective Förster radius of $19 \mathrm{~nm}$ was estimated for the $\mathrm{MoS}_{2}$ acceptor when using a nanoemitter with a bandgap of $\sim 2 \mathrm{eV}$. Yet, the Förster radius for an $\mathrm{MoS}_{2}$ acceptor is highly sensitive to the emission color of the donor since the absorption spectrum of $\mathrm{MoS}_{2}$ exhibits sharp excitonic absorption peaks at around 620 and $660 \mathrm{~nm}$. Thus, the spectral overlap term can be easily altered by using donors emitting at different spectral range. Also, monolayer $\mathrm{MoSe}_{2}$ was studied and shown to have large Förster radius $(\sim 15 \mathrm{~nm})$, similar to that of monolayer $\mathrm{MoS}_{2} \cdot{ }^{[27]}$ Strong dipole-dipole coupling and a large interaction range in TMDC monolayers, similar to graphene, have been attributed to strong light-matter interactions in these materials. As compared to their bulk counterparts, which commonly have an indirect bandgap, TMDC monolayers have a direct bandgap with large oscillator strength. Ref. [28] studied exciton transfer into a monolayer $\mathrm{SnS}_{2}$ from colloidal quantum dots. Different from TMDCs, monolayer $\mathrm{SnS}_{2}$ still has an indirect bandgap; 
thus, it has limited light absorption. In a monolayer $\mathrm{SnS}_{2}-$ quantum dot hybrid, the Förster radius was estimated to be ca. $5 \mathrm{~nm}$, suggesting its inferior exciton sink capability compared to the TMDCs and graphene.

Distance scaling of FRET into 2D materials has been a subject of interest. Theoretical studies have predicted $d^{-4}$ dependence due to the thin, planar nature of the acceptor. ${ }^{[20,93,101]}$ Lately, several experimental studies suggested strong evidence for $d^{-4}$ distance scaling, ${ }^{[22,23,27]}$ confirming theoretical expectations. These studies employed time-resolved and steady-state fluorescence spectroscopy to assess fluorescence decay rates and steady-state photoluminescence quenching factors as a function of the donor-acceptor separation distance. Smooth dielectric layers such as $\mathrm{MgO},{ }^{[22]} \mathrm{TiO}_{2},{ }^{[23]} \mathrm{HfO}_{2},{ }^{[26]} \mathrm{PMMA},{ }^{[102]}$ and $\mathrm{h}-\mathrm{BN}^{[27]}$ were employed to controllably tune the separation distance. Recently, Berciaud et al. compared the distance scaling of FRET into a single-layer graphene from spherical-like colloidal quantum dots and plate-like colloidal quantum wells. For a quantum dot donor, the FRET rate was found to scale with a $d^{-4}$ distance dependence (see Figure $4 \mathrm{a}$ ). In the case of colloidal quantum well donors, a slight deviation from $d^{-4}$ scaling was observed at short distances, which has been attributed to the larger lateral extent of the free excitons in the nanoplatelets, leading to a deviation from classical dipole emitter approximation. ${ }^{[22]}$ Few studies reported different distance dependencies such as $d^{-2.5[30]}$ and $d^{-2} \cdot{ }^{[24,25]}$ However, these unexpected scaling terms could simply arise due to the unexpected experimental conditions (e.g., non-smooth spacer layers) or potential charge transfer pathways.

One noteworthy difference between graphene and $\mathrm{MoS}_{2}$ was found on their dependence of energy transfer efficiency and rate as a function of their layer thicknesses. Prins et al. reported that as the number of $\mathrm{MoS}_{2}$ layers increases, FRET becomes less efficient. In contrast, Ref. [87] demonstrated that FRET into graphene becomes stronger as the graphene layer thickness is increased. A recent study compared FRET kinetics in both graphene and $\mathrm{MoS}_{2}$ as a function of layer thicknesses using the same donor (CdSe/ZnS quantum dots emitting at $2.1 \mathrm{eV})$. Figure $4 \mathrm{~b}$ shows the photoluminescence decay lifetime of the quantum dots on bare $\mathrm{SiO}_{2}$ (dashed line), $\mathrm{MoS}_{2} /$ $\mathrm{SiO}_{2}$ (red triangles), and graphene/ $\mathrm{SiO}_{2}$ (blue squares) samples, where the layer thickness of the 2D materials was varied from 1 to 25 monolayers. The intrinsic photoluminescence decay lifetime of the quantum dots on bare $\mathrm{SiO}_{2}$ was measured to be $\approx 5 \mathrm{~ns}$. When the same quantum dots are on either singlelayer $\mathrm{MoS}_{2}$ or single-layer graphene, the photoluminescence lifetime becomes considerably shorter ( $300 \mathrm{ps})$ due to efficient FRET into the 2D materials as reported earlier. Interestingly, as the layer thickness of $\mathrm{MoS}_{2}$ is increased, the photoluminescence lifetime of the quantum dots is observed to elongate, suggesting that the FRET efficiency decreases. In contrast, the photoluminescence lifetime of the donor becomes shorter as
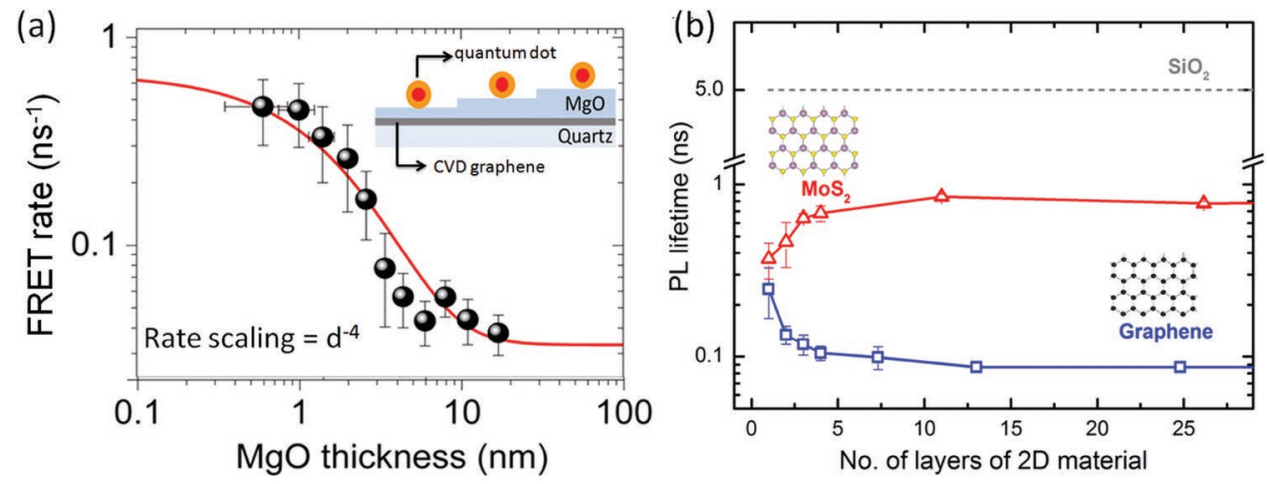

(c)
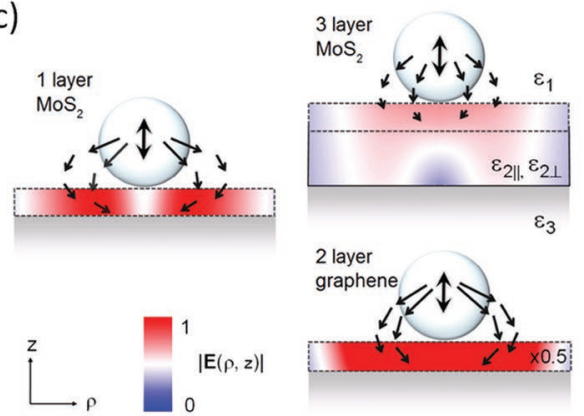

Figure 4. (a) Distance scaling of FRET rate into single layer from graphene $\mathrm{CdSe} / \mathrm{CdS}$ quantum dot. FRET rate into graphene scales with $d^{-4}$, where $d$ is the separation distance between graphene and quantum dot controlled by the thickness of dielectric spacer (MgO). Reproduced with permission. ${ }^{[22]}$ Copyright 2015, American Chemical Society. (b) Photoluminescence lifetime of the CdSe/ZnS quantum dot donor on MoS 2 (red triangles) and graphene (blue squares) as a function of the 2D layer thickness. As $\mathrm{MoS}_{2}$ layer thickness increases, decay lifetime of the quantum dots becomes longer, suggesting the suppression of FRET. As graphene layer thickness increases, decay lifetime of the quantum dots become shorter, suggesting that FRET grows stronger. The dashed line indicates the decay lifetime of the quantum dots on the bare $\mathrm{SiO}_{2}$ layer. (c) Near-field electric field distribution in single-layer and tri-layer $\mathrm{MoS}_{2}$, and bilayer graphene due to an oscillating transition dipole (i.e., quantum dot). Reproduced with permission:[88] Copyright 2016; American Chemical Society. 
the number of graphene layers is increased, suggesting that FRET efficiency effectively increases.

To understand and explain the observed differences between $\mathrm{MoS}_{2}$ and graphene, classical CPS (Chance, Prock, and Silbey) theory ${ }^{[52]}$ was employed, which revealed that screening effects are strikingly different between graphene and $\mathrm{MoS}_{2}$ due to the considerable difference of their complex dielectric functions. $\mathrm{MoS}_{2}$ is a highly polarizable material due to its large dielectric constant. Thus, as the layer thickness is increased, the electric field strength at the top $\mathrm{MoS}_{2}$ layer (which is closest to the nanoemitters) is strongly reduced (see Figure 4c) compared to a single-layer $\mathrm{MoS}_{2}$. In contrast, screening effect is not dominant for graphene due to its smaller real permittivity. Thus, increasing the layer thickness of graphene leads to a stronger absorption; in turn, this results in increased FRET rates. Overall, acceptor materials with less relative polarizability (e.g., graphene, GaSe and silicon) are expected to exhibit increasing FRET rates as the material thickness is increased. On the other hand, materials such as $\mathrm{MoS}_{2}, \mathrm{WS}_{2}$, and InAs will show FRET that becomes weaker as the layer thickness is increased. ${ }^{[88]}$ Furthermore, Gartstein et al. theoretically investigated and compared both macroscopic analyses by considering an acceptor slab having a complex dielectric function (i.e., CPS theory) and direct modeling, which considered discrete acceptor layers as polarizable point dipoles. ${ }^{[101,103]}$ In both models, non-additivity (non-monotonic behavior) in FRET efficiencies is observed as a function of the acceptor layer thickness, especially when the polarizability of the acceptor layer is relatively high (e.g., $\varepsilon^{\prime}>7$ and $\varepsilon^{\prime \prime}=0.2$ ). Overall, counter-intuitive trends observed in the nonradiative energy transfer efficiencies as a function of the 2D acceptor materials layer thicknesses can be accounted for by the electric field screening effect due to large polarizatibility of the acceptor layers. Thus, complex dielectric function of the acceptor materials should be investigated in more detail to efficiently tailor exciton flow at the nanoscale.

(a)
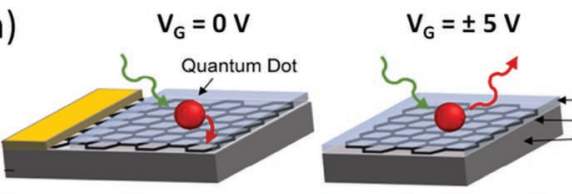

(b)

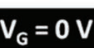

$\mathrm{V}_{\mathrm{G}}= \pm 5 \mathrm{~V}$
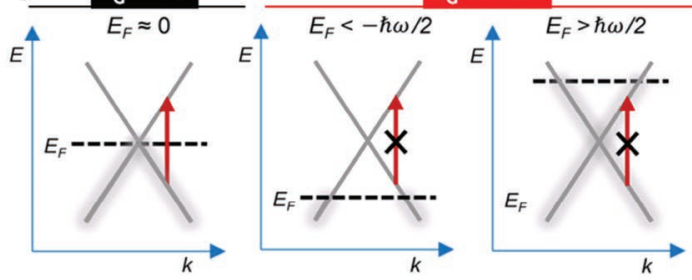

Another interesting observation when using 2D materials as an exciton sink is that blinking properties of the quantum dot donors are altered when they are coupled together. Previously, single quantum dots on graphene were found to blink less compared to those on bare $\mathrm{SiO}_{2}$ substrate. ${ }^{[22,87,90,104]}$ This was understood by the fact that strong FRET rate leads to competition with the internal charge-trapping mechanisms, which are essentially responsible for the blinking (fluorescence intermittency) of the colloidal nanocrystals. ${ }^{[105]}$ Therefore, thanks to large FRET rates, excitons can be efficiently transferred before nonradiative trapping could occur. On the other hand, in 2D material-quantum dot hybrids that exhibit weak FRET rates, no difference was observed on the blinking properties of the quantum dots compared to reference samples. ${ }^{[28,106,107]}$

Another advantage of 2D materials is the ease of controlling their photophysical properties through electrical means such as electrical gating. ${ }^{108]}$ Through electron filling of graphene, its Fermi level can be easily altered; therefore, interband transitions can be manipulated. In the last few years, several reports demonstrated that FRET from a nanoemitter into $2 \mathrm{D}$ materials could be turned on and off by electrical control on demand. ${ }^{[29,102,109]}$ Ref. [102] demonstrated that through electrostatic doping, FRET from single quantum dots to graphene could be reversibly inhibited. This was possible thanks to the modulation of the Fermi level of graphene via electron filling. Figure 5a shows the proposed back-gated graphene-quantum dot hybrid device. When the gate voltage is zero, the Fermi level $\left(E_{F} \approx 0\right)$ is approximately at the crossing point between conduction and valence bands. By applying gate voltage, it is possible to shift the Fermi level up (and down) by doping with electrons (holes). If $E_{F}>\frac{h v}{2}\left(\right.$ or $\left.E_{F}<-\frac{h v}{2}\right)$, then interband electronic transition at the photon energy $(h v)$ will be disallowed (see Figure 5b). Therefore, this would block FRET from a nearby donor having a bandgap of $h v$. Ref. [102] employed

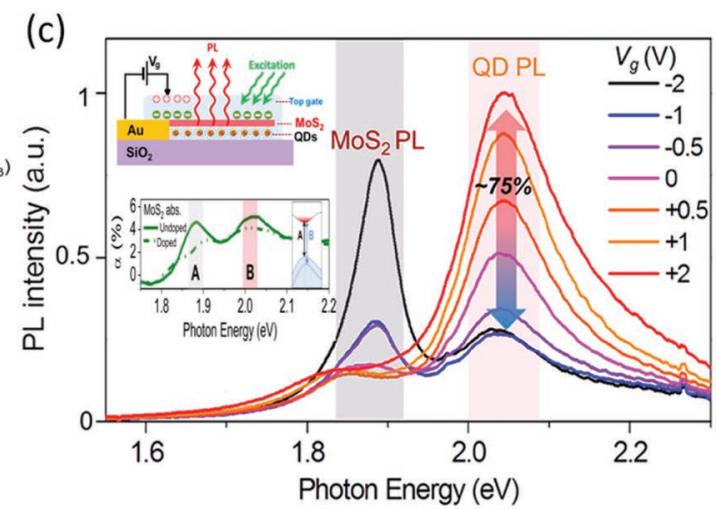

Figure 5. (a) Schematic of a back-gated graphene-quantum dot hybrid device. When $V_{G}=0 \mathrm{~V}$, FRET from quantum into graphene is allowed, thus quantum dot emission is strongly quenched. When $V_{G}= \pm 5 \mathrm{~V}$, electrons (or holes) dope graphene such that interband transitions are blocked, thus FRET is also blocked and quantum dot emission is recovered. (b) Energy band diagram demonstrating the modulation of the Fermi level of graphene via electronic gating. When $V_{G}=0 \mathrm{~V}, E_{\mathrm{F}} \approx 0$ and interband transition at the photon energy of the donor (red arrow) is allowed. When $V_{\mathrm{G}}= \pm 5 \mathrm{~V}$, then $E_{F}>\frac{h v}{2}$ or $E_{F}<-\frac{h v}{2}$, interband transition at the photon energy of the donor is not allowed, thus, FRET is blocked. Panels (b) and (c) are reproduced with permission. ${ }^{[102]}$ Copyright 2014, American Chemical Society. (c) Steady state photoluminescence spectra of the monolayer MoS ${ }_{2}-$ quantum dot hybrid device under different gate voltages. Quantum dot emission could be modulated by $75 \%$ due to modulation of FRET rate into MoS 2 . The top-left inset shows the device structure. The bottom-left inset shows the modulation of the absorption (solid for $V_{\mathrm{G}}=0 \mathrm{~V}$, dashed for $V_{\mathrm{G}} \neq 0 \mathrm{~V}$ ) of single-layer $\mathrm{MoS}_{2}$ under electronic gating. Reproduced with permission. ${ }^{[29]}$ Copyright 2015, American Chemical Society. 
$\mathrm{PbS}$ quantum dots having a bandgap in the range of $0.9-1.3 \mathrm{eV}$, where photoluminescence of the quantum dots could be modulated with a ratio larger than 2.3 folds by the gate-induced doping of graphene. Based on a similar approach, Koppens et al. also demonstrated electrical control of near-field energy transfer from excited erbium ions $(\sim 0.83 \mathrm{eV})$ to graphene by a rate modulation ratio of more than $3 .^{[109]}$

In addition to graphene, the $\mathrm{MoS}_{2}$ monolayer was proposed for electrical control of the FRET. ${ }^{[2]} \mathrm{MoS}_{2}$ exhibited suppressed absorption at its excitonic peaks under electrostatic gating. Therefore, the reduced absorption could be used to suppress the spectral overlap integral between the donor emission and the $\mathrm{MoS}_{2}$ absorption. In this work, a 5-fold modulation of FRET rate from $\mathrm{CdSe} / \mathrm{CdS}$ quantum dots into $\mathrm{MoS}_{2}$ was realized. Figure $5 c$ shows the steady-state photoluminescence spectra of the electrically gated $\mathrm{MoS}_{2}$-quantum dot hybrid device. Steady state photoluminescence emission of the quantum dots could be modified by $75 \%$. One advantage of $\mathrm{MoS}_{2}$ compared to graphene is that FRET from the nanoemitters with a bandgap in the visible range could be controlled because the excitonic absorption of $\mathrm{MoS}_{2}$ is in the visible range. As compared to graphene-based devices, $\mathrm{MoS}_{2}$ devices could also operate at smaller applied voltages. Although the on/off contrast ratio of the current proof-of-concept devices are limited (up to 5-folds), the concept of active FRET control will be exciting to develop quantum emitters that can be turned on and off on demand.

Although exciton transferring nanoemitter-graphene hybrid systems are exciting for light harvesting, a significant limitation arises because transferred excitons into graphene cannot efficiently contribute to the photocurrent generation due to ultrafast thermalization of the hot electron (and hot hole) in graphene. Previously, energy transfer from nanoemitters into a metal surface was studied and the transferred electron-hole pairs were considered to be lost within the electron bath of the metal due to fast thermalization of the hot carriers. ${ }^{[110]}$ Similarly, hot carrier relaxation in graphene typically takes place at ultrafast time scales (e.g., sub-picosecond), ${ }^{[111]}$ thus, resonantly transferred excitation energy is most probably lost to heating before it could be contributed to the photocurrent. As a potential solution to this problem in graphene, Ref. [112] recently reported an ultrafast photocurrent sensing scheme based on ultrafast photoconductive switches (e.g., Auston switches ${ }^{[113]}$ ). Closely separated striplines that act as an antenna on graphene were used for the ultrafast photoconductive detection and photocurrent extraction. Electron-hole pairs created in the graphene (either directly created or transferred via FRET) were swept out with the applied bias across two striplines. This generated an electromagnetic transient response in the stripline, which is sensed by a pump-probe scheme. The measured transient photocurrent revealed the contribution from the transferred electron-hole pairs from the nitrogen vacancy centers into the graphene. Resonantly transferred electron-hole pairs could be harvested and photocurrents on the order of $\mathrm{pA}$ level could be generated.

In addition, TMDC monolayers, which are semiconductors with a finite bandgap, are more suitable for harvesting the transferred excitons for photodetection because they have relatively long excited state lifetime. In TMDCs, the transferred electron and hole relax to the band edge and stay stable as the exciton for few ns, allowing enough time for photocurrent extraction, yet strong exciton binding energies in TMDC monolayers may cause difficulties for exciton dissociation. To this end, heterostructured TMDC layers having Type-II band alignment are promising for enhanced photocurrent generation and photovoltaics. ${ }^{[114]}$

In contrast to exciton transfer, charge transfer into 2D materials also created exciting opportunities for photosensing devices. ${ }^{[92,115]}$ Generally, core-only quantum dots have been employed, such as $\mathrm{PbS}$ quantum dots, because charge transfer is impeded in core/shell quantum dots. In addition to graphene, charge transfer has been observed from $\mathrm{PbS}$ quantum dots into reduced graphene oxide. ${ }^{[116]}$ Furthermore, $\mathrm{MoS}_{2}-\mathrm{PbS}$ quantum dot hybrids also accomplished efficient photodetection performance. ${ }^{[15]}$

\section{Efficient Exciton Transport Using Colloidal Nanoplatelets}

Recently, colloidal $\mathrm{Cd} X(X=\mathrm{Se}, \mathrm{S}$, and $\mathrm{Te})$ nanoplatelets have emerged as an exceptional addition to the class of semiconductor nanocrystals. The colloidal nanoplatelets possess atomically flat surfaces while their vertical thickness is only a discrete number of $\mathrm{CdX}$ monolayers. The lateral size of the nanoplatelets is typically larger than the exciton Bohr radius. Therefore, effectively one-dimensional quantum confinement is observed, which makes them colloidal analogues of epitaxial quantum wells. The nanoplatelets have exhibited superior optical properties compared to those of conventional nanocrystals (e.g., quantum dots). ${ }^{[10,117]}$ Due to very tight confinement in one dimension and strong dielectric screening, electron and hole are strongly bound with an exciton binding energy in excess of $200 \mathrm{meV}^{[118]}$ This leads to very large oscillator strength ${ }^{[119]}$ in the nanoplatelets, allowing them to bear large linear ${ }^{[71,120]}$ and nonlinear ${ }^{[121,122]}$ absorption cross-section together with fast radiative recombination rates. ${ }^{[10]}$ Also, there is no inhomogeneous size broadening in the nanoplatelet ensembles due to their atomically flat surfaces and fixed vertical thicknesses, which are also denoted by discrete number (i.e., monolayers) of the repeating lattice planes. ${ }^{[123,124]}$ Thanks to their advantageous properties, the nanoplatelets have become highly promising for optoelectronics with remarkable performance in solution-processed lasers, ${ }^{[125]}$ light-emitting diodes, ${ }^{[126]}$ and photodetectors. ${ }^{[127]}$

The potential for strong light-matter interactions in the nanoplatelets, similar to 2D semiconductors, make them exciting candidates for efficient exciton transfer at the nanoscale. In Ref. [31], we uncovered ultrafast exciton transfer among CdSe nanoplatelets in their face-to-face stacked assemblies (see Figure 6), which form due to strong van der Waals forces. ${ }^{[128]}$ We controllably stacked the nanoplatelets that were dissolved in hexane by slowly adding an anti-solvent (ethanol). As face-to-face stacking occurs, photoluminescence intensity was strongly quenched and simultaneously fluorescence decays became faster, suggesting an overall increase in the nonradiative recombination rate in the nanoplatelet ensembles.

We accounted for these observations by ultraefficient FRETassisted exciton trapping in the nanoplatelets. We estimated a 

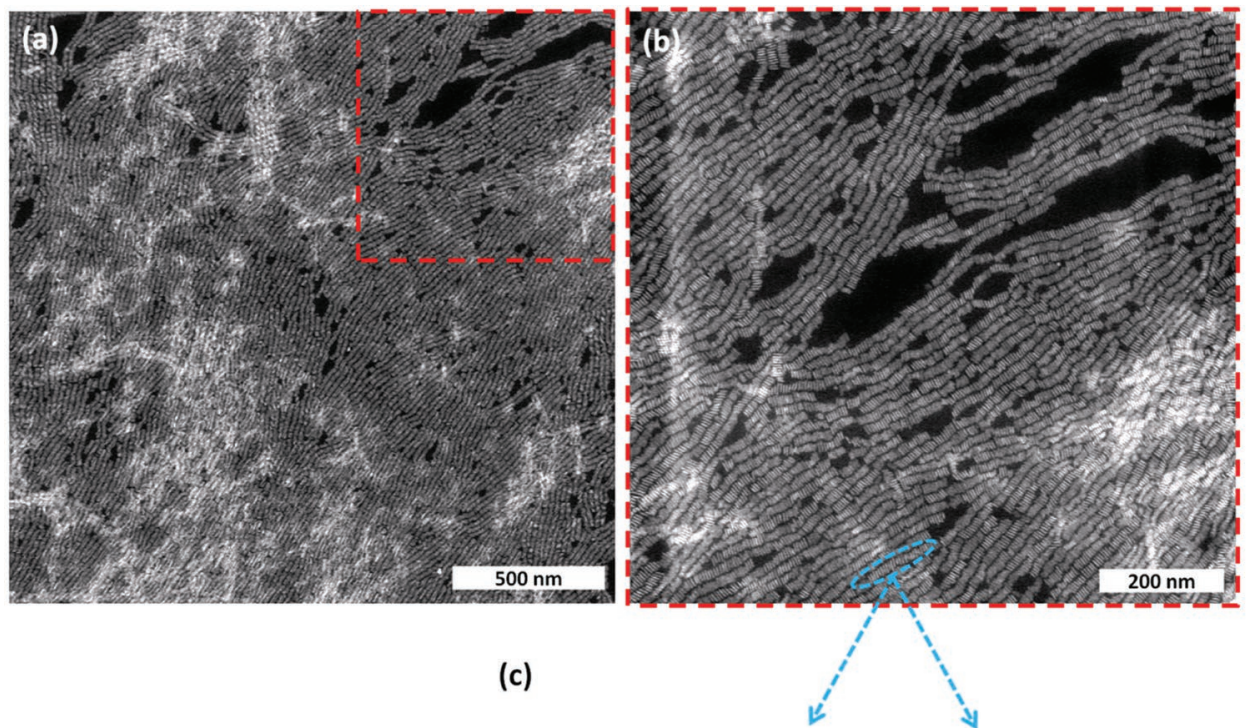

(c)

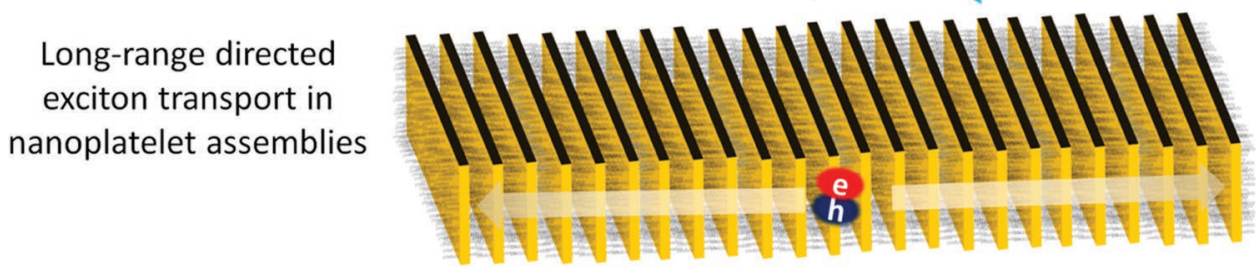

Figure 6. High-angle annular dark-field transmission electron microscopy (HAADF-STEM) image of highly ordered face-to-face stacked CdSe nanoplatelets with scale bars (a) $500 \mathrm{~nm}$ and (b) $200 \mathrm{~nm}$. The region in (b) is from the dashed area in (a). Highly ordered needle-like nanoplatelet stacks were achieved as shown by the representative schematic in (c). In the absence of non-emissive nanoplatelets, ultralong exciton diffusion length (>100 nm) is expected.

very large Förster radius of $13.5 \mathrm{~nm}$ (see Table 1) for the homoFRET between nanoplatelets having the same vertical thickness (four monolayer CdSe). This large Förster radius was enabled by the large linear absorption cross-section and very small Stokes shift in the nanoplatelets together with collinear orientation of the transition dipoles in their face-to-face stacked assemblies. The Förster radius for the nanoplatelet acceptors is almost two folds larger than that of the conventional quantum dot acceptors (see Table 1). Also, it is comparable to the largest reported Förster radius using 2D materials such as graphene and TMDC monolayers.

In the face-to-face assembled nanoplatelets, we predicted FRET efficiency as high as $99.9 \%$ with an ultrafast FRET lifetime of $3 \mathrm{ps}$ for the two neighboring nanoplatelets in the stacked assembly, which offers minimized separation distance ( $\approx 4.2 \mathrm{~nm}$ ) between the nanoplatelets. Previously, the fastest FRET lifetime among colloidal nanocrystals was reported $\approx 100$ ps. ${ }^{[72,129]}$ Thus, colloidal nanoplatelets stand out as ultrafast exciton scavengers among colloidal semiconductors. Moreover, we estimated an effective exciton diffusion length that is larger than $130 \mathrm{~nm} \cdot{ }^{[31]}$ The exciton diffusion would be also directed and anisotropic due to the needle-like geometry of the stacked assemblies (see Figure 6). Previously, exciton diffusion in the colloidal quantum dots was found to be limited by $\sim 20 \mathrm{~nm}$ due to sub-diffusive exciton transport ${ }^{[73]}$ arising from downhill transport in the inhomogeneously broadened density of states due to size polydispersity in the quantum dots. This had caused a strong red-shift of the emission due to homo-FRET in close-packed quantum dot films. ${ }^{[130]}$ In contrast, we did not observe a red-shift of the nanoplatelet emission because inhomogeneous broadening is substantially suppressed. Previously, perovskite films showed comparable exciton diffusion lengths in excess of $100 \mathrm{~nm} \cdot{ }^{[131]}$ Further studies based on nanoscale exciton diffusion visualization will be extremely useful in the nanoplatelet assemblies. ${ }^{[132,133]}$ Achievement of ordered, needle-like, stacked nanoplatelet films ${ }^{[134]}$ will be promising for the solution-processed solar cells, photodetectors, and artificial photosynthesis thanks to the potential of ultralong exciton diffusion length. In Figure 6, we show our results on achieving ordered needle-like nanoplatelet films on TEM grids via an air/ liquid self-assembly technique. ${ }^{[135]}$ Highly ordered nanoplatelet stacks with more than 100 nanoplatelets per stack could be achieved at relatively large areas.

On the other hand, we found that nanoplatelet ensembles contain a sub-population of poorly-emitting nanoplatelets due to the presence of surface traps in some of the nanoplatelets, ${ }^{[121]}$ which was also observed and reported by others. ${ }^{[136,137]}$ Theoretical modeling strongly suggested that ultraefficient FRET-assisted exciton trapping is the dominant mechanism of the photoluminescence quenching in face-to-face assembled nanoplatelets. Ultraefficient exciton transport among the nanoplatelets within the stack leads to an increased capturing rate for the non-emissive nanoplatelets. Figure 7 shows both face-to-face and side-by-side assembled nanoplatelets. In the face-to-face stacked assembly, ultraefficient exciton transfer among the nanoplatelets leads to increased exciton trapping 

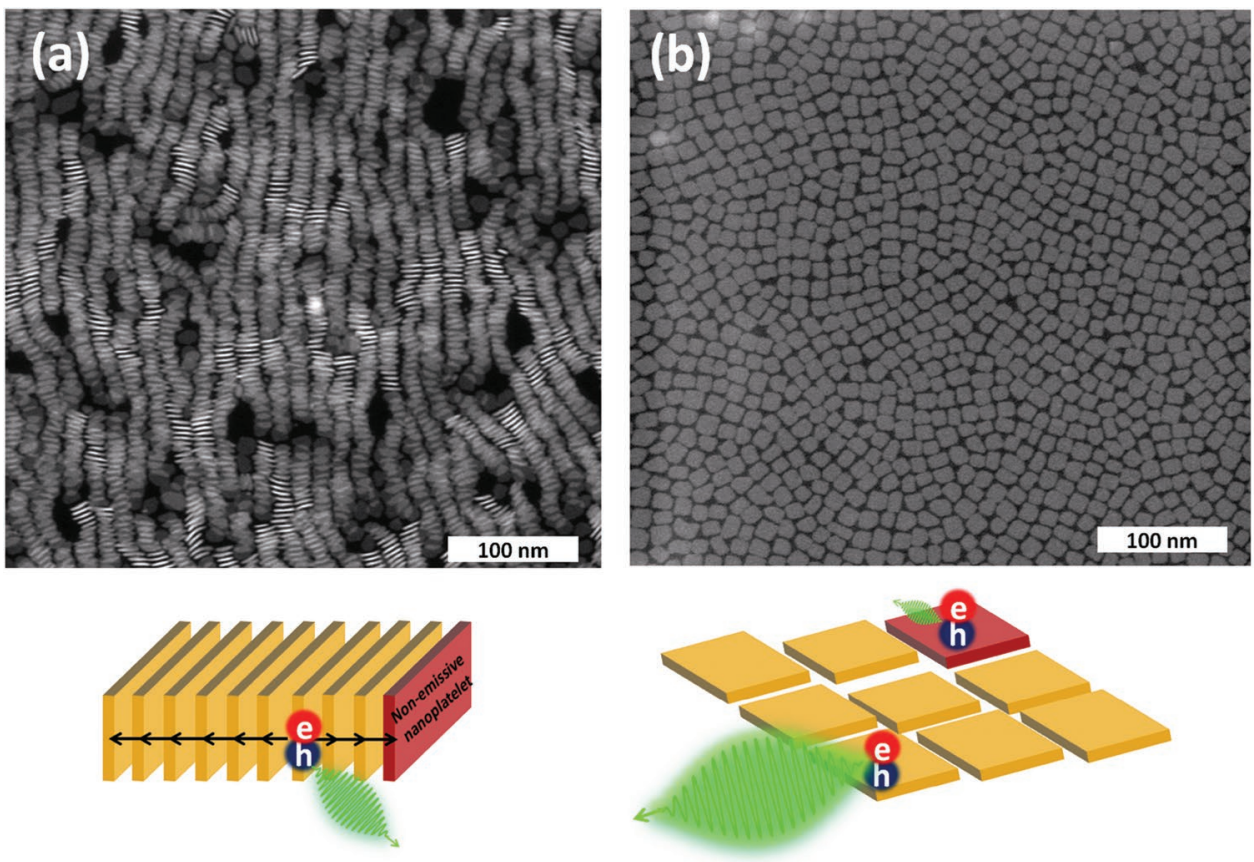

Figure 7. HAADF-STEM images of (a) face-to-face and (b) side-by-side stacked nanoplatelet assemblies realized by air/liquid self-assembly technique. (a) FRET-assisted exciton trapping by non-emissive nanoplatelet leads to strongly quenched emission. (b) Exciton trapping is suppressed as dipoledipole coupling is weaker due to increased separation distance between the nanoplatelets in the side-by-side stacked assemblies.

in the poorly emissive nanoplatelets. When exciton ends up in a poorly emissive nanoplatelet, exciton is nonradiatively quenched by either hole or electron trapping (see Figure 7a). In mosaic-like side-by-side assembled nanoplatelets, exciton transfer is not dominant because the donor-acceptor distance is large. Therefore, this assembly is more favorable to preserve the photoluminescence intensity in the presence of non-emissive nanoplatelets (see Figure $7 \mathrm{~b}$ ).

FRET-assisted exciton trapping in the face-to-face stacked nanoplatelets was also evidenced by studying temperaturedependent photoluminescence kinetics in face-to-face stacked nanoplatelets. ${ }^{[138]}$ In this recent work, we found that sparsely side-by-side stacked nanoplatelets exhibit considerably increased photoluminescence intensity as the temperature is decreased. This was previously attributed to the increased oscillator strength in the nanoplatelets at low temperatures, which increases their emission quantum yield. ${ }^{[10]}$ On the other hand, face-to-face stacked nanoplatelets showed 5-folds less increase as the temperature was decreased in the same range. Through theoretical modeling, we revealed that FRET-assisted exciton trapping becomes more efficient as oscillator strength is increased at low temperatures. Therefore, exciton trapping grows stronger at low temperatures in the face-to-face stacked nanoplatelets.

In addition to homo-FRET, hetero-FRET was also investigated among nanoplatelet ensembles. First, we demonstrated that FRET is possible from four monolayer- into five monolayer-thick CdSe nanoplatelets. ${ }^{[33]}$ In this work, we showed unambiguous evidence of FRET via time-resolved fluorescence and photoluminescence excitation spectra. Although we estimated a Förster radius of ca. $11 \mathrm{~nm}$ (see Table 1), we unexpectedly found that FRET efficiencies are limited by $60 \%$ (see Figure 8a). Through electron microscopy, we discovered that four- and five-monolayer CdSe nanoplatelet stack in their own populations due to their different lateral sizes (see the schematic in Figure 8a). Consequently, the effective distance between the donor and the acceptor is large, considerably limiting FRET. Thus, we could not achieve ultrafast FRET due to nanoscale morphology of the nanoplatelets. On the other hand, Talapin et al. were able to interstack four- and five-monolayerthick CdSe nanoplatelets together (see inset of Figure 8b). ${ }^{[139]}$ Thus, ultrafast FRET rates were measured using streak camera, exhibiting FRET rates as fast as 6 ps (see Figure 8b). This report is in excellent agreement with our prediction of 3 ps FRET rate in Ref. [31]. Moreover, the ultrafast FRET rates observed by Refs. [139] and [31] suggest that self-assembled nanoplatelets will be highly suitable for realizing efficient FRET in the multiexciton regime, which has not been possible before using conventional quantum dots. This will make the nanoplatelets exciting prospects for solar light harvesting.

In addition to nanoplatelet-nanoplatelet systems, we recently employed nanoplatelets as exciton harvesters from blue-emitting CdZnS/ZnS core/shell quantum dots. ${ }^{[32]}$ By assuming random dipole orientation due to isotropic donor quantum dots, we calculated an $\approx 9 \mathrm{~nm}$ Förster radius (see Table 1). Quantum dots and nanoplatelets mixed well together without phase segregation, allowing us to achieve FRET efficiencies as high as $\mathbf{9 0 \%}$ at room temperature (see Figure 9 a). an interesting feature of these hybrids was that they exhibited an exceptionally linear response for the FRET efficiencies as a function of temperature in the range of 300-50 K (Figure 9b). The dominant effect behind this linear FRET behavior was found to be the temperature-dependent PL QY of the donor quantum dots. ${ }^{[32]}$ 
(a)

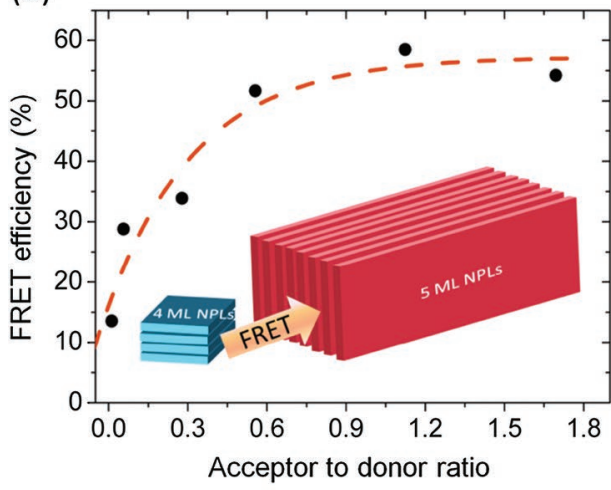

(b)

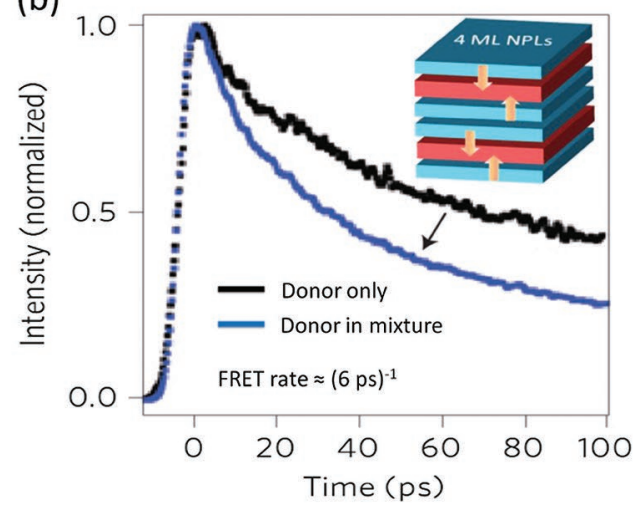

Figure 8. (a) FRET efficiency as a function of acceptor (five-monolayer) to donor (four-monolayer-thick CdSe nanoplatelet) molar ratio. FRET efficiencies were unexpectedly saturated around $60 \%$ due to nanoscale phase segregation arising from the self-stacking of the donor and acceptor on their own as shown by the cartoon at the bottom. Reproduced with permission. ${ }^{[3]}$ Copyright 2015, Royal Society of Chemistry. (b) Experimental evidence of ultra-fast FRET rate ( $\approx 6 \mathrm{ps}$ ) from four- to five-monolayer-thick CdSe nanoplatelets that are interstacked together as shown by the cartoon on top-right. Reproduced with permission. ${ }^{[139]}$ Copyright 2015, Nature Publishing Group.

Also, the change of the temperature-dependent spectral overlap played a minor role in the observed FRET efficiencies. Therefore, this hybrid system could be employed as non-contact temperature probes. The sensitivity of the quantum dot-nanoplatelet hybrid was found to be $3.2 \% \mathrm{~K}^{-1}$, which is comparable to the best report $\left(3.5 \% \mathrm{~K}^{-1[140]}\right)$ using a ratio metric sensing scheme. Here, our hybrid systems employed FRET efficiencies for temperature sensing, which can be characterized via timeresolved fluorescence techniques, thus allowing for increased robustness and reliability compared to ratio metric sensing that depend on emission intensities.

\section{Dexter Energy Transfer for Triplet-State Exciton Harvesting}

In the last decade, organic semiconductor-colloidal nanocrystal hybrids have been explored owing to their promising application in solar cells ${ }^{[141]}$ and light-emitting diodes. ${ }^{[142,143]} \mathrm{A}$ commonly studied excitonic process in the organic-nanocrystal hybrids is the spin-singlet exciton transfer. ${ }^{[144-147]}$ Exploiting organicnanocrystal hybrids that exhibit highly efficient exciton transfer, we developed hybrid LEDs, in which quantum dot emitters were dominantly pumped by exciton transfer by the organic semiconductor. ${ }^{[36]}$ Although these excitonically pumped hybrid LEDs make interesting alternatives to charge injection pumping, they are limited by the use of fluorescent organic semiconductors. Under free carrier injection, $3 / 4$ of the formed excitons in a fluorescent organic molecule are in spin-triplet state. Thus, it was not possible to harvest these triplet excitons via FRET because triplet states have almost zero oscillator strength.

Triplet-state exciton harvesting in organic-nanocrystal hybrids were addressed by employing phosphorescent small organic molecules, which have mixed singlet-triplet states due to strong intersystem crossing. As a result, FRET became feasible when using phosphorescent molecules as exciton donors, (a)

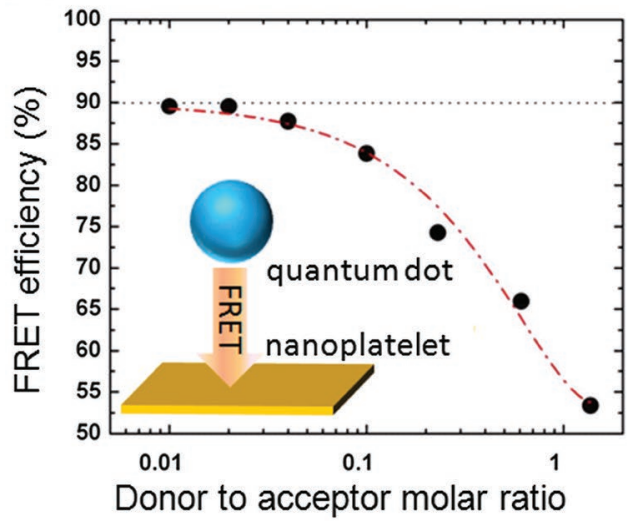

(b)

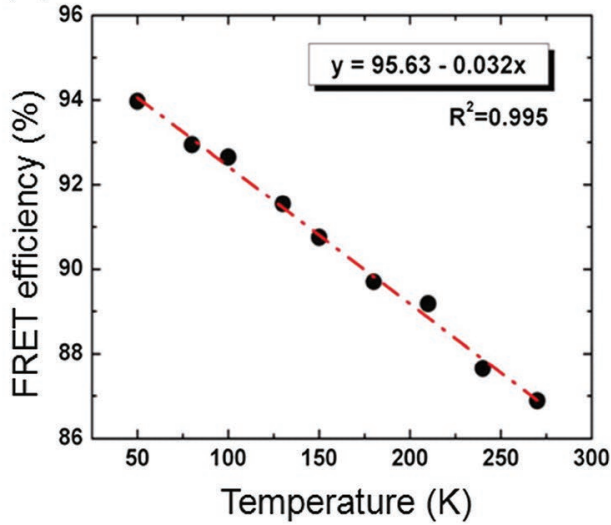

Figure 9. (a) FRET efficiency in colloidal quantum dot-nanoplatelet nanocomposites as a function of donor (quantum dot) to acceptor (nanoplatelet) molar ratio. (b) Temperature-dependent FRET efficiency in quantum dot-nanoplatelet nanocomposite. Panels (a) and (b) reproduced with permission. ${ }^{32}$ ] Copyright 2016, John Wiley \& Sons, Inc. 
which was revealed by time-resolved fluorescence and photoluminescence excitation measurements. ${ }^{[38,148]}$ Although FRET rates were slow $\left(k_{F R E T}^{-1} \approx 50 n s\right)$ in these hybrids, overall FRET efficiencies were found to be as high as $90 \%{ }^{[38]}$ due to an intrinsically slow radiative decay rate in the phosphorescent molecules. Also, relatively large Förster radius ( $6 \mathrm{~nm}$, see Table 1 ) was predicted for the cyan-emitting FIrpic phosphorescent donor-red-emitting CdSe/CdZnS core/shell quantum dots (see Table 1). We also deployed phosphorescent molecule-quantum dot hybrids in active LEDs to harvest both singlet and triplet excitons. Ir(ppy $)_{3}$ molecules were partially co-doped into an electron transport layer adjacent to the emissive quantum dot layer. ${ }^{[149]}$ Excitons formed in the co-doped layer due to leaking holes through the quantum dot film were recycled back into the emissive layer via FRET. This exciton recycling within hybrid LEDs resulted in a 6-fold increase in the external quantum efficiency of the devices while preserving an excellent spectral color purity (99\% from the quantum dots). ${ }^{[149]}$

More recently, spin-triplet excitons in small organic molecules (e.g., pentacene and tetracene) were shown to be efficiently transferred into colloidal quantum dots (e.g., $\mathrm{PbS}$ and $\mathrm{PbSe}$ ) via Dexter energy transfer. ${ }^{[150-152]}$ This nonradiative energy transfer was shown to achieve ultrafast transfer rates $(\approx \text { few } p s)^{[151]}$ with transfer efficiencies reaching almost unity. ${ }^{[152]}$ These studies further highlighted that colloidal quantum dots make highly efficient sensitizers to exploit ultraefficient multiexciton processes in organic semiconductors such as singlet exciton fission ${ }^{[153,154]}$ $\left(S_{1} \rightarrow 2 T_{1}\right)$ and triplet-triplet annihilation ${ }^{[155]}\left(2 T_{1} \rightarrow S_{1}\right)$. Thus, organic-nanocrystal hybrids are expected to pave the way for efficient-use molecular excitons to harness light more efficiently in solar cells or enhance light generation performance in hybrid LEDs and upconversion systems.
Figure 10 schematically exemplifies two schemes that deploy organic-nanocrystal hybrids for efficient light-harvesting and upconversion applications. In Figure 10a, a high energy photon absorption generates singlet exciton in the organic semiconductor. Through singlet exciton fission, two triplet excitons can be formed from a singlet exciton with exceptionally high efficiencies $(\approx 200 \%)$. ${ }^{[154]}$ These triplet excitons can then be transferred to colloidal quantum dots via Dexter energy transfer. Afterwards, the quantum dots can either emit a longer wavelength photon or can transfer the excitation energy into another semiconductor (e.g., silicon) via nonradiative energy transfer, as previously discussed. With this scheme, light harvesting with internal quantum efficiencies exceeding $100 \%$ can be achieved owing to the exploitation of the multiexciton generation. ${ }^{[156]}$

Figure 10b schematically illustrates a hybrid upconversion scheme that employs NIR-IR-absorbing quantum dots and visible-emitting organic molecules. Colloidal quantum dots, which have a smaller bandgap than the first singlet exciton energy of the organic molecule, can transfer its exciton into the organic molecule via Dexter energy transfer. As a consequence, triplet excitons are generated in the organic molecule, which can form higher-energy singlet excitons via triplet-triplet annihilation (i.e., triplet fusion). Singlet excitons in the organic molecule will emit visible photons. Thus, efficient IR-to-visible upconversion can be achieved by using quantum dots having a tunable bandgap in IR region.

Organic semiconductor-colloidal nanocrystal hybrids that perform upconversion were recently realized using smallbandgap $\mathrm{Cd}$ - and $\mathrm{Pb}$-chalcogenide quantum dots by transferring their excitons into the triplet states of larger-bandgap organic molecules (e.g., diphenylanthracene, rubrene and 9-anthracenecarboxylic acid, naphthyl). ${ }^{[157-160]}$ Previously, IR-to-visible (a)

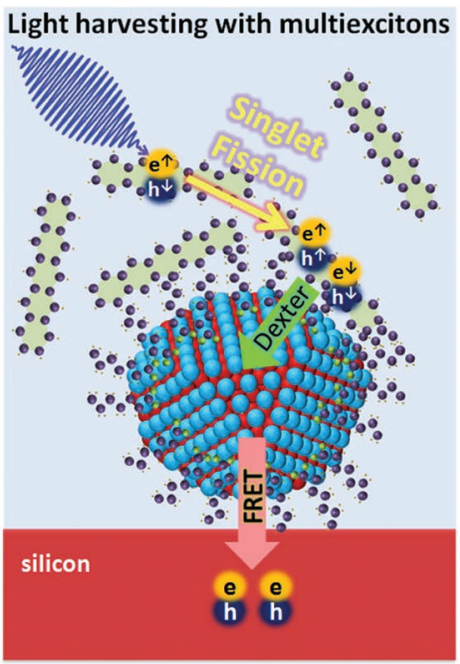

(b)

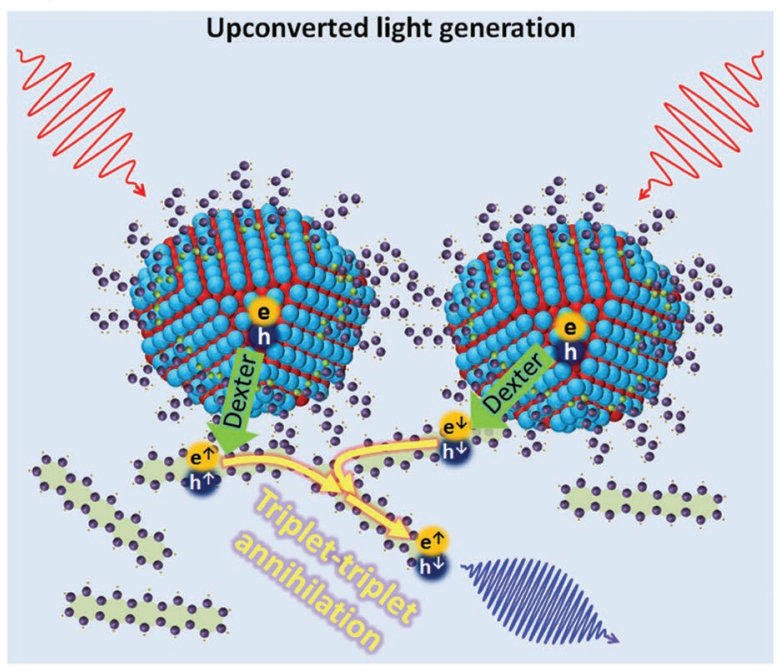

Figure 10. (a) A sensitization scheme for silicon solar cells based on organic semiconductor-colloidal nanocystal hybrids. A singlet exciton generated by absorption of high energy photons leads to the generation of two triplet state excitons via singlet exciton fission. Triplet excitons are harvested by the quantum dots through Dexter energy transfer. Excitons in the quantum dots can be transferred into silicon via FRET or RET, increasing the overall power conversion efficiencies. (b) An upconversion scheme based on organic semiconductor-colloidal nanocrystal hybrids. Excitons generated by IR photons in the quantum dots are resonantly transferred into molecules as triplet excitons via Dexter energy transfer. Stored triplet excitons can perform triplet-triplet annihilation to form high energy singlet excitons that emit photons in the visible range. 
upconversion has been very limited due to lack of efficient sensitizers having a bandgap in the IR. ${ }^{[158]}$ Most of the conventional organic sensitizers have a bandgap in the visible to NIR range and lanthanide-doped crystals have very limited spectral coverage in the IR. ${ }^{[158]}$ Thus, PbS quantum dots with a tunable bandgap in the NIR-IR $(\approx 1.2-1.4 \mathrm{eV})$ were demonstrated to be promising. Figure 11a shows the upconverted emission spectrum of dibenzotetraphenylperiflanthene (DBP). $\mathrm{PbS}$ quantum dots having three different bandgaps (first excitonic absorption peaks at 850, 960, and $1010 \mathrm{~nm}$ ) were used as NIR-IR sensitizers. $\mathrm{PbS}$ quantum dots transferred excitons into rubrene, where triplet-triplet annihilation takes place. Singlet excitons in the rubrene are harvested by DBP molecules, which have a very high photoluminescence quantum yield. Figure $11 \mathrm{~b}$ depicts both the Dexter energy transfer rate $\left(k_{\mathrm{ET}}\right)$ from CdSe quantum dots into anthracene ligands and the the upconversion quantum yield. As smaller anthracene-based ligands (e.g., 9ACA) are used, both Dexter energy transfer rate and upconversion efficiency increases compared to the longer ligands (e.g., CPPA). Among Pb-chalcogenide quantum dots, PbSe was shown to outperform PbS. ${ }^{[158]}$ This has been attributed to the larger exciton Bohr radius of $\mathrm{PbSe}(47 \mathrm{~nm})$ as compared to that of $\mathrm{PbS}$ $(18 \mathrm{~nm}) \cdot{ }^{[7]}$ Thus, a larger extent of the charge wavefuncions in PbSe quantum dots is expected to increase electron exchange coupling (i.e., Dexter energy transfer). Similarly, smaller quantum dots were found to boost the upconversion yield compared to quantum dots that have a larger size. ${ }^{[161]}$

Through optimization of the size and ligands of the quantum dots and molecular emitter system, upconversion quantum efficiencies exceeded $2.1 \%$ for the IR-to-visible upconversion systems. ${ }^{[162]}$ Moreover, visible-to-visible upconversion using CdSe quantum dot sensitizers led to a large quantum efficiency of $14 \% .{ }^{[163]}$ The best reports for visible-to-visible and IR-tovisible upconversion quantum efficiencies were $32 \%$ and $5 \%$, respectively. ${ }^{[164]}$ Considering that organic-nanocrystal hybrids have only been investigated in the last year, these systems with efficient solid-state upconversion possibilities ${ }^{[160]}$ will offer huge potential for IR-sensitization of inorganic solar cells and bio-imaging.

\section{Conclusions and Future Outlook}

In this feature article, we focused on hybrid excitonic systems and their striking near-field energy transfer interactions for enhanced exciton harvesting in light detection and generation applications. Specifically, we reviewed exciton transfer phenomena in composite systems of colloidal nanocrystalsnanostructured semiconductors, nanoemitters-2D semiconductors, organic semiconductors-colloidal nanocrystals, and atomically flat colloidal nanoplatelets as well as our recent results on their self-assembly. These hybrid systems are empowered by strong light-matter interactions in the emerging nanomaterials, leading to extraordinary and ultrahigh-efficiency excitonic interactions. Tailoring controlled exciton flow in the designer nanostructured hybrid systems will enable ultraefficiency in artificial excitonic systems, offering exciting opportunities for numerous applications such as optoelectronics, photovoltaics, sensing, and bio-imaging. Here, we foresee important future research directions in these excitonic hybrids that will positively impact their optoelectronic applications.

\subsection{Multiexciton Harvesting via Ultrafast Exciton Transfer}

Multiexciton generation and harvesting has been considered as one of the promising schemes to overcome the ShockleyQueisser limit in conventional solar cells. ${ }^{[47]}$ Nanoemitters such as colloidal quantum dots and organic semiconductors were shown to realize multiexciton generation per absorbed photon $\left(h v>2 E_{g}\right)$. However, multiexcitons, especially in colloidal nanocrystals, are very short-lived (50-100 ps) due to Auger recombination, which annihilates multiexcitons nonradiatively. Because of this, it has proven difficult to extract multiexcitons
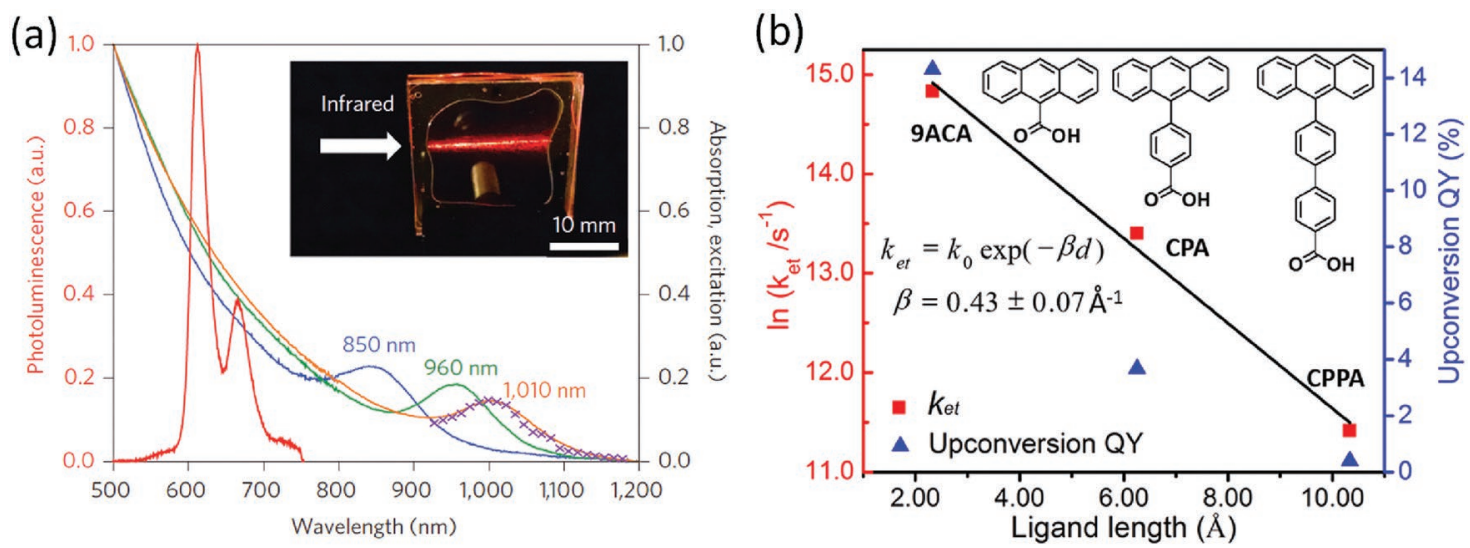

Figure 11. (a) Upconverted luminescence spectrum from DBP molecules in the red spectral range sensitized by PbS quantum dots absorbing in the NIR-IR region (having first excitonic absorption peaks at 850,960 , and $1010 \mathrm{~nm}$ ). The inset shows the red upconverted emission in the solid-state PbS quantum dot-rubrene: DBP hybrid upon IR excitation from the edge of the sample. Reproduced with permission. ${ }^{[160]}$ Copyright 2016 , Nature Publishing Group. (b) Dexter energy transfer rate (squares) and upconversion quantum efficiency (triangles) shown for CdSe quantum dot-anthracene hybrids. Shorter anthracene ligand (e.g., 9ACA) results in large energy transfer rate and higher upconversion quantum efficiency. Longer ligands suppress Dexter energy transfer and upconversion. Reproduced with permission. ${ }^{[163]}$ Copyright 2016, American Chemical Society. 
from nanoemitters for their effective use in light harvesting. Recent studies reported very long range and strong dipoledipole coupling via employing emerging nanomaterials such as graphene, ${ }^{[23]}$ TMDC monolayers ${ }^{[30]}$ and Cd-chalcogenide nanoplatelets. ${ }^{[31,139]}$ These materials allow for ultrafast FRET processes (a few ps), which, using multiexcitons formed in nearby nanoemitters, can be efficiently transferred before losing them to Auger recombination processes. Therefore, exploiting multiexcitons in nanoemitters via ultrafast FRET will allow for enhanced energy conversion in solar cells and also in optical gain systems with reduced lasing thresholds. In addition, localized surface plasmon coupled energy transfer can be employed to boost FRET efficiencies by increasing the effective distance range of the FRET. ${ }^{[165-168]}$

\subsection{Long-Range Exciton Diffusion in Self-Assembling Colloidal Nanoplatelets and other Emerging Nanocrystals}

Face-to-face assembled colloidal nanoplatelets have shown strong promise for long range exciton diffusion thanks to the prevailing ultraefficient homo-FRET. However, presence of nonemissive nanoplatelets in the nanoplatelet ensembles leads to enhanced exciton trapping and thus severe photoluminescence quenching. Recently, advanced heterostructures of the nanoplatelets were developed, including core/crown/shell, ${ }^{[169]}$ core/ multi-crown, and core/multi-shell. These nanoplatelets exhibit increased surface passivation along with increased stability and high quantum efficiencies. Thus, well-passivated nanoplatelets are expected to suppress exciton trapping and reach potentially ultralong exciton diffusion in their self-assembled solid films.

Additionally, recently introduced cesium lead halide perovskite nanocrystals ${ }^{[170]}$ and their atomically flat nanoplatelets ${ }^{[171]}$ exhibiting strong light-matter interactions are expected to empower strong dipole-dipole coupling and potentially allow long range exciton diffusion similar to organic lead halide perovskite films. ${ }^{[131]}$ Self-assembled nanocrystals with long exciton diffusion (>100 $\mathrm{nm}$ ) will be exciting for artificial photosynthesis, catalysis, solar cells, and photodetectors.

\subsection{Light-Generation Using 2D Materials-Nanoemitters Structures}

To date, 2D materials such as graphene and TMDC monolayers have been intensively investigated for their exciton sink capabilities, exhibiting unprecedentedly strong dipole-dipole coupling with exceptionally large Förster radius (>10 nm). Lately, research on light-emission properties of $2 \mathrm{D}$ materials resulted in reports of near-unity emission quantum yields ${ }^{[172]}$ and efficient LEDs, ${ }^{[82]}$ as well as lasers. ${ }^{[173]}$ Thus, utilizing 2D materials as efficient exciton generators and pumps will be exciting for light generation applications. Excitons that are efficiently formed in the 2D materials under electrical injection could be nonradiatively transferred to nearby nanoemitters such as nanoplatelets with very high color purity (emission full-width at half-maximum $\approx 8 \mathrm{~nm}$ at room temperature) to realize hybrid 2D light-emitting diodes. These excitonically pumped 2D material-nanocrystal hybrid LEDs will be promising because they will minimize intrinsic waveguiding losses in OLEDs and also potentially accomplish optically and/or electrically pumped lasing.

\section{Acknowledgement}

The authors would like to express thanks for the financial support from Singapore National Research Foundation under the programs of NRFNRFI-2016-08, NRF-RF-2009-09, NRF-CRP-6-2010-02 and the Science and Engineering Research Council, Agency for Science, Technology and Research (A*STAR) of Singapore (project Nos. 0921010057 and 112120 2009), EU-FP7 Nanophotonics4Energy NoE, and TUBITAK EEEAC 114F326, 114E449,114E410 and 115E679. H.V.D. acknowledges support from ESF-EURYI and TUBA-GEBIP.

Received: July 1, 2016

Revised: August 15, 2016

Published online: October 12, 2016

[1] S. P. Fasol, S. Nakamura, The Blue Laser Diode: The Complete Story, 2nd ed., Springer-Verlag, Berlin 2000.

[2] E. F. Schubert, Light-Emitting Diodes, 2nd ed., Cambridge University Press, New York, 2006.

[3] C. B. Murray, D. J. Norris, M. G. Bawendi, J. Am. Chem. Soc. 1993, $115,8706$.

[4] N. S. Sariciftci, L. Smilowitz, A. J. Heeger, F. Wudl, Science 1992, 258, 1474.

[5] C. W. Tang, S. A. VanSlyke, Appl. Phys. Lett. 1987, 51, 913.

[6] K. S. Novoselov, A. K. Geim, S. V. Morozov, D. Jiang, M. I. Katsnelson, I. V. Grigorieva, S. V. Dubonos, A. A. Firsov, Nature 2005, 438, 197.

[7] B. Guzelturk, P. L. H. Martinez, Q. Zhang, Q. Xiong, H. Sun, X. W. Sun, A. O. Govorov, H. V. Demir, Laser Photon. Rev. 2014, 8, 73.

[8] M. G. Bawendi, M. L. Steigerwald, L. E. Brus, Annu. Rev. Phys. Chem. 1990, 41, 477

[9] A. Ramasubramaniam, Phys. Rev. B 2012, 86, 115409.

[10] S. Ithurria, M. D. Tessier, B. Mahler, R. P. S. M. Lobo, B. Dubertret, A. L. Efros, Nat. Mater. 2011, 10, 936.

[11] M. Achermann, M. A. Petruska, S. Kos, D. L. Smith, D. D. Koleske, V. I. Klimov, Nature 2004, 429, 642.

[12] B. E. Hardin, E. T. Hoke, P. B. Armstrong, J.-H. Yum, P. Comte, T. Torres, J. M. J. Fréchet, M. K. Nazeeruddin, M. Grätzel, M. D. McGehee, Nat. Photonics 2009, 3, 406.

[13] A. L. Rogach, T. A. Klar, J. M. Lupton, A. Meijerink, J. Feldmann, J. Mater. Chem. 2009, 19, 1208.

[14] Y. Shirasaki, G. J. Supran, M. G. Bawendi, V. Bulović, Nat. Photonics 2013, 7, 933

[15] A. Nurmikko, Nat. Nanotechnol. 2015, 10, 1001.

[16] T. Förster, Ann. Phys. 1948, 437, 55.

[17] D. L. Dexter, J. Chem. Phys. 1953, 21, 836.

[18] R. M. Clegg, Chapter 1 Förster resonance energy transfer-FRET what is it, why do it, and how it's done, in Laboratory Techniques in Biochemistry and Molecular Biology, FRET and Film Techniques), (Eds: P. C. van der Vliet, S. Pillai), Vol. 33, Elsevier B. V., Amsterdam 2009, 1-57.

[19] P. L. Hernández-Martínez, A. O. Govorov, H. V. Demir, J. Phys. Chem. C 2014, 118, 4951.

[20] P. L. Hernández-Martínez, A. O. Govorov, H. V. Demir, J. Phys. Chem. C 2013, 117, 10203.

[21] J. R. Lakowicz, Principles of Fluorescence Spectroscopy, Springer, Boston, MA 2006.

[22] F. Federspiel, G. Froehlicher, M. Nasilowski, S. Pedetti, A. Mahmood, B. Doudin, S. Park, J.-O. Lee, D. Halley, B. Dubertret, P. Gilliot, S. Berciaud, Nano Lett. 2015, 15, 1252. 
[23] L. Gaudreau, K. J. Tielrooij, G. E. D. K. Prawiroatmodjo, J. Osmond, F. J. G. de Abajo, F. H. L. Koppens, Nano Lett. 2013, 13, 2030.

[24] Y.-J. Yu, K. S. Kim, J. Nam, S. R. Kwon, H. Byun, K. Lee, J.-H. Ryou, R. D. Dupuis, J. Kim, G. Ahn, S. Ryu, M.-Y. Ryu, J. S. Kim, Nano Lett. 2015, 15, 896.

[25] T. N. Lin, L. T. Huang, G. W. Shu, C. T. Yuan, J. L. Shen, C. A. J. Lin W. H. Chang, C. H. Chiu, D. W. Lin, C. C. Lin, H. C. Kuo, Opt. Lett. 2013, 38, 2897.

[26] A. Yeltik, G. Kucukayan-Dogu, B. Guzelturk, S. Fardindoost, Y. Kelestemur, H. V. Demir, J. Phys. Chem. C 2013, 117, 25298.

[27] K. M. Goodfellow, C. Chakraborty, K. Sowers, P. Waduge, M. Wanunu, T. Krauss, K. Driscoll, A. N. Vamivakas, Appl. Phys. Lett. 2016, 108, 021101

[28] H. Zang, P. K. Routh, Y. Huang, J.-S. Chen, E. Sutter, P. Sutter, M. Cotlet, ACS Nano 2016, 10, 4790.

[29] D. Prasai, A. R. Klots, A. Newaz, J. S. Niezgoda, N. J. Orfield C. A. Escobar, A. Wynn, A. Efimov, G. K. Jennings, S. J. Rosenthal, K. I. Bolotin, Nano Lett. 2015, 15, 4374.

[30] F. Prins, A. J. Goodman, W. A. Tisdale, Nano Lett. 2014, 14, 6087.

[31] B. Guzelturk, O. Erdem, M. Olutas, Y. Kelestemur, H. V. Demir, ACS Nano 2014, 8, 12524.

[32] M. Olutas, B. Guzelturk, Y. Kelestemur, K. Gungor, H. V. Demir, Adv. Funct. Mater. 2016, 26, 2891

[33] B. Guzelturk, M. Olutas, S. Delikanli, Y. Kelestemur, O. Erdem H. V. Demir, Nanoscale 2015, 7, 2545.

[34] H. M. Nguyen, O. Seitz, D. Aureau, A. Sra, N. Nijem, Y. N. Gartstein, Y. J. Chabal, A. V. Malko, Appl. Phys. Lett. 2011, 98, 161904.

[35] M. Dutta, L. Thirugnanam, P. Van Trinh, N. Fukata, ACS Nano 2015, 9, 6891.

[36] B. Guzelturk, P. L. Hernandez Martinez, V. K. Sharma, Y. Coskun, V. Ibrahimova, D. Tuncel, A. O. Govorov, X. W. Sun, Q. Xiong, H. V. Demir, P. L. Hernandez-Martinez, Nanoscale 2014, 6, 11387

[37] R. S. Deshpande, V. Bulović, S. R. Forrest, Appl. Phys. Lett. 1999, 75,888 .

[38] B. Guzelturk, P. L. Hernandez Martinez, D. Zhao, X. W. Sun, H. V. Demir, J. Phys. Chem. C 2014, 118, 25964.

[39] D. L. Dexter, J. Lumin. 1979, 18-19, 779.

[40] V. M. Agranovich, Y. N. Gartstein, M. Litinskaya, Chem. Rev. 2011 $111,5179$.

[41] B. O'Regan, M. Grätzel, Nature 1991, 353, 737.

[42] Y. X. Liu, M. A. Summers, C. Edder, J. M. J. Frechet, M. D. McGehee, Adv. Mater. 2005, 17, 2960.

[43] S. Lu, Z. Lingley, T. Asano, D. Harris, T. Barwicz, S. Guha, A. Madhukar, Nano Lett. 2009, 9, 4548

[44] G. H. Carey, A. L. Abdelhady, Z. Ning, S. M. Thon, O. M. Bakr, E. H. Sargent, Chem. Rev. 2015, 115, 12732.

[45] B. Guzelturk, E. Mutlugun, X. Wang, K. L. Pey, H. V. Demir, Appl. Phys. Lett. 2010, 97, 093111.

[46] M. A. Green, Third Generation Photovoltaics: Advanced Solar Energy Conversion, Vol. 10, Springer-Verlag, Berlin 2006.

[47] M. C. Beard, K. P. Knutsen, P. Yu, J. M. Luther, Q. Song, W. K. Metzger, R. J. Ellingson, A. J. Nozik, Nano Lett. 2007, 7, 2506.

[48] T. Trupke, M. A. Green, P. Würfel, J. Appl. Phys. 2002, 92, 1668.

[49] B. S. Richards, Sol. Energy Mater. Sol. Cells 2006, 90, 1189.

[50] E. Mutlugun, I. M. Soganci, H. V. Demir, Opt. Express 2008, 16, 3537.

[51] E. Garnett, P. Yang, Nano Lett. 2010, 10, 1082.

[52] R. R. Chance, A. Prock, R. Silbey, in Advances in Chemical Physics, (Eds: S. A. Rice, I. Prigogine), John Wiley \& Sons, New York 1978, pp. 1-65.

[53] A. P. Alivisatos, M. F. Arndt, S. Efrima, D. H. Waldeck, C. B. Harris, J. Chem. Phys. 1987, 86, 6540.
[54] P. M. Whitmore, A. P. Alivisatos, C. B. Harris, Phys. Rev. Lett. 1983, 50, 1092.

[55] S. Lu, A. Madhukar, Nano Lett. 2007, 7, 3443.

[56] S. Chanyawadee, R. T. Harley, M. Henini, D. V. Talapin, P. G. Lagoudakis, Phys. Rev. Lett. 2009, 102, 1.

[57] S. Chanyawadee, R. T. Harley, D. Taylor, M. Henini, A. S. Susha, A. L. Rogach, P. G. Lagoudakis, Appl. Phys. Lett. 2009, 94, 233502.

[58] T. Aqua, R. Naaman, A. Aharoni, U. Banin, Y. Paltiel, Appl. Phys. Lett. 2008, 92.

[59] S. Nizamoglu, E. Sari, J. H. Baek, I. H. Lee, H. V. Demir, Phys. Status Solidi RRL 2010, 4, 178

[60] A. Dorn, D. B. Strasfeld, D. K. Harris, H. S. Han, M. G. Bawendi, ACS Nano 2011, 5, 9028.

[61] A. Yeltik, B. Guzelturk, P. L. Hernandez-Martinez, A. O. Govorov, H. V. Demir, ACS Nano 2013, 7, 10492.

[62] I. Moreels, Y. Justo, G. Rainò, T. Stöferle, Z. Hens, R. F. Mahrt, Adv. Opt. Mater. 2014, 2, 126.

[63] E. Mutlugun, P. L. Hernandez-Martinez, C. Eroglu, Y. Coskun T. Erdem, V. K. Sharma, E. Unal, S. K. Panda, S. G. Hickey, N. Gaponik, A. Eychmüller, H. V. Demir, Nano Lett. 2012, 12, 3986.

[64] M. Stavola, D. L. Dexter, R. S. Knox, Phys. Rev. B 1985, 31, 2277.

[65] H. M. Nguyen, O. Seitz, W. Peng, Y. N. Gartstein, Y. J. Chabal, A. V. Malko, ACS Nano 2012, 6, 5574.

[66] M. T. Nimmo, L. M. Caillard, W. De Benedetti, H. M. Nguyen, O. Seitz, Y. N. Gartstein, Y. J. Chabal, A. V. Malko, ACS Nano 2013 7, 3236.

[67] W. Peng, S. Sampat, S. M. Rupich, B. Anand, H. M. Nguyen, D. Taylor, B. E. Beardon, Y. N. Gartstein, Y. J. Chabal, A. V Malko Nanoscale 2015, 7, 8524

[68] S. Hoang, A. Ashraf, M. D. Eisaman, D. Nykypanchuk, C.-Y. Nam, Nanoscale 2015

[69] M. Dutta, N. Fukata, Nano Energy 2015, 11, 219.

[70] W. W. Yu, L. Qu, W. Guo, X. Peng, Chem. Mater. 2003, 15, 2854.

[71] A. W. Achtstein, A. Antanovich, A. Prudnikau, R. Scott, U. Woggon, M. Artemyev, J. Phys. Chem. C 2015, 119, 20156.

[72] S. Crooker, J. Hollingsworth, S. Tretiak, V. Klimov, Phys. Rev. Lett. 2002, 89, 186802

[73] G. M. Akselrod, F. Prins, L. V. Poulikakos, E. M. Y. Lee, M. C. Weidman, A. J. Mork, A. P. Willard, V. Bulović, W. A. Tisdale, Nano Lett. 2014 14, 3556.

[74] A. Yeltik, B. Guzelturk, P. Ludwig Hernandez-Martinez, S. Akhavan, H. Volkan Demir, Appl. Phys. Lett. 2013, 103, 261103.

[75] W. J. I. De Benedetti, M. T. Nimmo, S. M. Rupich, L. M. Caillard, Y. N. Gartstein, Y. J. Chabal, A. V. Malko, Adv. Funct. Mater. 2014, 24, 5002.

[76] T. Franzl, T. A. Klar, S. Schietinger, A. L. Rogach, J. Feldmann, Nano Lett. 2004, 4, 1599.

[77] A. Ruland, C. Schulz-Drost, V. Sgobba, D. M. Guldi, Adv. Mater. 2011, 23, 4573.

[78] I. J. Kramer, L. Levina, R. Debnath, D. Zhitomirsky, E. H. Sargent, Nano Lett. 2011, 11, 3701.

[79] F. Xu, X. Ma, C. R. Haughn, J. Benavides, M. F. Doty, S. G. Cloutier, ACS Nano 2011, 5, 9950.

[80] A. H. Castro Neto, F. Guinea, N. M. R. Peres, K. S. Novoselov, A. K. Geim, Rev. Mod. Phys. 2009, 81, 109.

[81] G. Fiori, F. Bonaccorso, G. Iannaccone, T. Palacios, D. Neumaier A. Seabaugh, S. K. Banerjee, L. Colombo, Nat. Nanotechnol. 2014, 9, 768.

[82] Q. H. Wang, K. Kalantar-Zadeh, A. Kis, J. N. Coleman, M. S. Strano, Nat. Nanotechnol. 2012, 7, 699.

[83] S. Z. Butler, S. M. Hollen, L. Cao, Y. Cui, J. A. Gupta, H. R. Gutiérrez, T. F. Heinz, S. S. Hong, J. Huang, A. F. Ismach, E. Johnston-Halperin, M. Kuno, V. V. Plashnitsa, R. D. Robinson, 
R. S. Ruoff, S. Salahuddin, J. Shan, L. Shi, M. G. Spencer, M. Terrones, W. Windl, J. E. Goldberger, ACS Nano 2013, 7, 2898.

[84] D. Jariwala, V. K. Sangwan, L. J. Lauhon, T. J. Marks, M. C. Hersam, ACS Nano 2014, 8, 1102

[85] Y. Liu, X. Dong, P. Chen, Chem. Soc. Rev. 2012, 41, 2283.

[86] Y. Shao, J. Wang, H. Wu, J. Liu, I. A. Aksay, Y. Lin, Electroanalysis 2010, 22, 1027.

[87] Z. Chen, S. Berciaud, C. Nuckolls, T. F. Heinz, L. E. Brus, ACS Nano 2010, 4, 2964.

[88] A. Raja, A. Montoya-Castillo, J. Zultak, X.-X. Zhang, Z. Ye, C. Roquelet, D. A. Chenet, A. M. van der Zande, P. Huang, S. Jockusch, J. Hone, D. R. Reichman, L. E. Brus, T. F. Heinz, Nano Lett. 2016, 16, 2328.

[89] E. Malic, H. Appel, O. T. Hofmann, A. Rubio, J. Phys. Chem. C 2014, $118,9283$.

[90] O. A. Ajayi, N. C. Anderson, M. Cotlet, N. Petrone, T. Gu, A. Wolcott, F. Gesuele, J. Hone, J. S. Owen, C. W. Wong, Appl. Phys. Lett. 2014, 104, 171101.

[91] S. Sampat, T. Guo, K. Zhang, J. A. Robinson, Y. Ghosh, K. P. Acharya, H. Htoon, J. A. Hollingsworth, Y. N. Gartstein, A. V. Malko, ACS Photonics 2016, 3, 708

[92] G. Konstantatos, M. Badioli, L. Gaudreau, J. Osmond, M. Bernechea, F. P. Garcia de Arquer, F. Gatti, F. H. L. Koppens, Nat. Nanotechnol. 2012, 7, 363.

[93] R. S. Swathi, K. L. Sebastian, J. Chem. Phys. 2009, 130, 086101

[94] I. V. Lightcap, P. V. Kamat, J. Am. Chem. Soc. 2012, 134, 7109.

[95] Y. Wang, Z. Li, J. Wang, J. Li, Y. Lin, Trends Biotechnol. 2011, 29, 205.

[96] S. Jander, A. Kornowski, H. Weller, Nano Lett. 2011, 11, 5179.

[97] E. Shafran, B. D. Mangum, J. M. Gerton, Nano Lett. 2010, 10, 4049.

[98] J. Tisler, T. Oeckinghaus, R. J. Stöhr, R. Kolesov, R. Reuter, F. Reinhard, J. Wrachtrup, Nano Lett. 2013, 13, 3152.

[99] J. Kim, L. J. Cote, F. Kim, J. Huang, J. Am. Chem. Soc. 2010, 132, 260.

[100] S. Li, M. L. Steigerwald, L. E. Brus, ACS Nano 2009, 3, 1267.

[101] J. M. Gordon, Y. N. Gartstein, J. Phys. Condens. Matter 2013, 25, 425302.

[102] J. Lee, W. Bao, L. Ju, P. J. Schuck, F. Wang, A. Weber-Bargioni, Nano Lett. 2014, 14, 7115

[103] J. M. Gordon, Y. N. Gartstein, J. Opt. Soc. Am. B 2014, 31, 2029.

[104] B. Rogez, H. Yang, E. Le Moal, S. Lévêque-Fort, E. Boer-Duchemin, F. Yao, Y.-H. Lee, Y. Zhang, K. D. Wegner, N. Hildebrandt, A. Mayne, G. Dujardin, J. Phys. Chem. C 2014, 118, 18445

[105] P. Spinicelli, S. Buil, X. Quélin, B. Mahler, B. Dubertret, J. P. Hermier, Phys. Rev. Lett. 2009, 102, 136801.

[106] S. Sampat, N. S. Karan, T. Guo, H. Htoon, J. A. Hollingsworth, A. V. Malko, ACS Photonics 2015, 2, 1505.

[107] N. J. Orfield, J. R. McBride, F. Wang, M. R. Buck, J. D. Keene, K. R. Reid, H. Htoon, J. A. Hollingsworth, S. J. Rosenthal, ACS Photonics, 2016, 10, 1960.

[108] F. Wang, Y. Zhang, C. Tian, C. Girit, A. Zettl, M. Crommie, Y. R. Shen, Science 2008, 320, 206

[109] K. J. Tielrooij, L. Orona, A. Ferrier, M. Badioli, G. Navickaite, S. Coop, S. Nanot, B. Kalinic, T. Cesca, L. Gaudreau, Q. Ma, A. Centeno, A. Pesquera, A. Zurutuza, H. de Riedmatten, P. Goldner, F. J. García de Abajo, P. Jarillo-Herrero, F. H. L. Koppens, Nat. Phys. 2015, 11, 281.

[110] D. H. Waldeck, A. P. Alivisatos, C. B. Harris, Surf. Sci. 1985, 158, 103

[111] J. C. Johannsen, S. Ulstrup, F. Cilento, A. Crepaldi, M. Zacchigna, C. Cacho, I. C. E. Turcu, E. Springate, F. Fromm, C. Raidel, T. Seyller, F. Parmigiani, M. Grioni, P. Hofmann, Phys. Rev. Lett. 2013, 111, 027403
[112] A. Brenneis, L. Gaudreau, M. Seifert, H. Karl, M. S. Brandt, H. Huebl, J. A. Garrido, F. H. L. Koppens, A. W. Holleitner, Nat. Nanotechnol. 2015, 10, 135

[113] D. H. Auston, A. M. Johnson, P. R. Smith, J. C. Bean, Appl. Phys. Lett. 1980, 37, 371 .

[114] C.-H. Lee, G.-H. Lee, A. M. van der Zande, W. Chen, Y. Li, M. Han, X. Cui, G. Arefe, C. Nuckolls, T. F. Heinz, J. Guo, J. Hone, P. Kim, Nat. Nanotechnol. 2014, 9, 676

[115] D. Kufer, I. Nikitskiy, T. Lasanta, G. Navickaite, F. H. L. Koppens, G. Konstantatos, Adv. Mater. 2015, 27, 176

[116] B. Martín-García, A. Polovitsyn, M. Prato, I. Moreels, J. Mater. Chem. C 2015, 3, 7088 .

[117] E. Lhuillier, S. Pedetti, S. Ithurria, B. Nadal, H. Heuclin, B. Dubertret, Acc. Chem. Res. 2015, 48, 22.

[118] R. Benchamekh, N. a. Gippius, J. Even, M. O. Nestoklon, J.-M. Jancu, S. Ithurria, B. Dubertret, A. L. Efros, P. Voisin, Phys. Rev. B 2014, 89, 035307.

[119] A. Naeem, F. Masia, S. Christodoulou, I. Moreels, P. Borri, W. Langbein, Phys. Rev. B 2015, 91, 121302.

[120] A. Yeltik, S. Delikanli, M. Olutas, Y. Kelestemur, B. Guzelturk, H. V. Demir, J. Phys. Chem. C 2015, 119, 26768

[121] M. Olutas, B. Guzelturk, Y. Kelestemur, A. Yeltik, S. Delikanli, H. V. Demir, ACS Nano 2015, 9, 5041

[122] R. Scott, A. W. Achtstein, A. Prudnikau, A. Antanovich, S. Christodoulou, I. Moreels, M. Artemyev, U. Woggon, Nano Lett., 2015, 15, 4985

[123] C. She, I. Fedin, D. S. Dolzhnikov, A. Demortière, R. D. Schaller, M. Pelton, D. V Talapin, D. Richard, Nano Lett. 2014, 14, 2772.

[124] M. D. Tessier, C. Javaux, I. Maksimovic, V. Loriette, B. Dubertret, L. T. Kunneman, H. Heuclin, Y. V. Aulin, F. C. Grozema, J. M. Schins, L. D. A. Siebbeles, ACS Nano 2013, 4, 3574.

[125] B. Guzelturk, Y. Kelestemur, M. Olutas, S. Delikanli, H. V. Demir, ACS Nano 2014, 8, 6599.

[126] Z. Chen, B. Nadal, B. Mahler, H. Aubin, B. Dubertret, Adv. Funct. Mater. 2014, 24, 295.

[127] E. Lhuillier, J.-F. Dayen, D. O. Thomas, A. Robin, B. Doudin, B. Dubertret, Nano Lett. 2015, 15, 1736.

[128] B. Abécassis, M. D. Tessier, P. Davidson, B. Dubertret, Nano Lett. 2014, 14, 710.

[129] T. Franzl, D. S. Koktysh, T. A. Klar, A. L. Rogach, J. Feldmann, N. Gaponik, Appl. Phys. Lett. 2004, 84, 2904.

[130] C. Kagan, C. Murray, M. Nirmal, M. Bawendi, Phys. Rev. Lett. 1996, 76, 1517.

[131] G. Xing, N. Mathews, S. Sun, S. S. Lim, Y. M. Lam, M. Grätzel, S. Mhaisalkar, T. C. Sum, Science 2013, 342, 344.

[132] G. M. Akselrod, P. B. Deotare, N. J. Thompson, J. Lee, W. A. Tisdale, M. A. Baldo, V. M. Menon, V. Bulović, Nat. Commun. 2014, 5, 3646.

[133] S. B. Penwell, L. D. S. Ginsberg, N. S. Ginsberg, J. Phys. Chem. Lett. 2015, 6, 2767.

[134] S. Jana, P. Davidson, B. Abécassis, Angew. Chem., Int. Ed. 2016, 55,9371

[135] E. V. Shevchenko, D. V. Talapin, N. A. Kotov, S. O'Brien, C. B. Murray, Nature 2006, 439, 55.

[136] L. T. Kunneman, J. M. Schins, S. Pedetti, H. Heuclin, F. C. Grozema, A. J. Houtepen, B. Dubertret, L. D. A. Siebbeles, Nano Lett. 2014, 14, 7039.

[137] F. T. Rabouw, J. C. Van Der Bok, P. Spinicelli, B. Mahler, M. Nasilowski, S. Pedetti, B. Dubertret, D. Vanmaekelbergh, Nano Lett. 2016, 16, 2047.

[138] O. Erdem, M. Olutas, B. Guzelturk, Y. Kelestemur, H. V. Demir, J. Phys. Chem. Lett. 2016, 7, 548

[139] C. E. Rowland, I. Fedin, H. Zhang, S. K. Gray, A. O. Govorov, D. V. Talapin, R. D. Schaller, Nat. Mater. 2015, 14, 484. 
[140] X. Rao, T. Song, J. Gao, Y. Cui, Y. Yang, C. Wu, B. Chen, G. Qian, J. Am. Chem. Soc. 2013, 135, 15559.

[141] W. U. Huynh, J. J. Dittmer, A. P. Alivisatos, Science 2002, 295, 2425.

[142] S. Coe, W.-K. Woo, M. Bawendi, V. Bulović, Nature 2002, 420, 800.

[143] V. L. Colvin, M. C. Schlamp, A. P. Alivisatos, Nature 1994, 370, 354.

[144] M. Anni, L. Manna, R. Cingolani, D. Valerini, A. Cretí, M. Lomascolo, Appl. Phys. Lett. 2004, 85, 4169.

[145] B. Guzelturk, H. V. Demir, J. Phys. Chem. Lett. 2015, 6, 2206.

[146] P. T. K. Chin, R. A. M. Hikmet, R. A. J. Janssen, J. Appl. Phys. 2008, 104, 013108.

[147] G. Jiang, A. S. Susha, A. A. Lutich, F. D. Stefani, J. Feldmann, A. L. Rogach, ACS Nano 2009, 3, 4127.

[148] P. O. Anikeeva, C. F. Madigan, S. A. Coe-Sullivan, J. S. Steckel, M. G. Bawendi, V. Bulović, Chem. Phys. Lett. 2006, 424, 120.

[149] E. Mutlugun, B. Guzelturk, A. P. Abiyasa, Y. Gao, X. W. Sun, H. V. Demir, J. Phys. Chem. Lett. 2014, 5, 2802.

[150] A. Khetubol, S. Van Snick, A. Hassinen, E. Fron, Y. Firdaus, L. Pandey, C. C. David, K. Duerinckx, W. Dehaen, Z. Hens, M. Van der Auweraer, J. Appl. Phys. 2013, 113, 083507.

[151] M. Tabachnyk, B. Ehrler, S. Gélinas, M. L. Böhm, B. J. Walker K. P. Musselman, N. C. Greenham, R. H. Friend, A. Rao, Nat. Mater. 2014, 13, 1033.

[152] N. J. Thompson, M. W. B. Wilson, D. N. Congreve, P. R. Brown, J. M. Scherer, T. S. Bischof, M. Wu, N. Geva, M. Welborn, T. Van Voorhis, V. Bulović, M. G. Bawendi, M. A. Baldo, Nat. Mater. 2014, 13, 1039

[153] M. B. Smith, J. Michl, Chem. Rev. 2010, 110, 6891.

[154] M. W. B. Wilson, A. Rao, J. Clark, R. S. S. Kumar, D. Brida, G. Cerullo, R. H. Friend, J. Am. Chem. Soc. 2011, 133, 11830.

[155] T. N. Singh-Rachford, F. N. Castellano,Coord. Chem. Rev. 2010, $254,2560$.

[156] O. E. Semonin, J. M. Luther, S. Choi, H.-Y. Chen, J. Gao, A. J. Nozik, M. C. Beard, Science 2011, 334, 1530.

[157] A. C. Samia, X. Chen, C. Burda, J. Am. Chem. Soc. 2003, 125 15736.

[158] Z. Huang, X. Li, M. Mahboub, K. M. Hanson, V. M. Nichols, H. Le, M. L. Tang, C. J. Bardeen, Nano Lett. 2015, 15, 5552.
[159] C. Mongin, S. Garakyaraghi, N. Razgoniaeva, M. Zamkov, F. N. Castellano, Science, 2016, 351, 369.

[160] M. Wu, D. N. Congreve, M. W. B. Wilson, J. Jean, N. Geva, M. Welborn, T. Van Voorhis, V. Bulovi, M. G. Bawendi, M. A. Baldo, Nat. Photonics 2015, 10, 31

[161] M. Mahboub, H. Maghsoudiganjeh, A. M. Pham, Z. Huang M. L. Tang, Adv. Funct. Mater. 2016.

[162] Z. Huang, D. E. Simpson, M. Mahboub, X. Li, M. L. Tang, Chem. Sci. 2016, 7, 4101.

[163] X. Li, Z. Huang, R. Zavala, M. L. Tang, J. Phys. Chem. Lett. 2016, 7, 1955.

[164] J. Zhou, Q. Liu, W. Feng, Y. Sun, F. Li,Chem. Rev. 2015, 115, 395.

[165] V. K. Komarala, A. L. Bradley, Y. P. Rakovich, S. J. Byrne, Y. K. Gun'ko, A. L. Rogach, Appl. Phys. Lett. 2008, 93, 123102.

[166] M. Lunz, V. A. Gerard, Y. K. Gun'ko, V. Lesnyak, N. Gaponik, A. S. Susha, A. L. Rogach, A. L. Bradley, Nano Lett. 2011, 11 3341.

[167] X. Zhang, C. A. Marocico, M. Lunz, V. A. Gerard, Y. K. Gun'ko, V. Lesnyak, N. Gaponik, A. S. Susha, A. L. Rogach, A. L. Bradley, ACS Nano 2012, 6, 9283.

[168] T. Ozel, P. L. Hernandez-Martinez, E. Mutlugun, O. Akin, S. Nizamoglu, I. O. Ozel, Q. Zhang, Q. Xiong, H. V. Demir, Nano Lett., 2013, 13, 3065.

[169] Y. Kelestemur, B. Guzelturk, O. Erdem, M. Olutas, K. Gungor, H. V. Demir, Adv. Funct. Mater. 2016, 26, 3570.

[170] L. Protesescu, S. Yakunin, M. I. Bodnarchuk, F. Krieg, R. Caputo, C. H. Hendon, R. X. Yang, A. Walsh, M. V. Kovalenko, Nano Lett. 2015, 15, 3692.

[171] L. Dou, A. B. Wong, Y. Yu, M. Lai, N. Kornienko, S. W. Eaton, A. Fu, C. G. Bischak, J. Ma, T. Ding, N. S. Ginsberg, L.-W. Wang A. P. Alivisatos, P. Yang, Science 2015, 349, 1518.

[172] M. Amani, D.-H. Lien, D. Kiriya, J. Xiao, A. Azcatl, J. Noh, S. R. Madhvapathy, R. Addou, S. KC, M. Dubey, K. Cho, R. M. Wallace, S.-C. Lee, J.-H. He, J. W. Ager, X. Zhang, E. Yablonovitch, A. Javey, Science 2015, 350, 1065.

[173] Y. Ye, Z. J. Wong, X. Lu, X. Ni, H. Zhu, X. Chen, Y. Wang, X. Zhang, Nat. Photonics 2015, 9, 733. 